\title{
Transcriptome and metabolite profiling analyses provide insight into volatile aroma compounds of the apple cultivar 'Ruixue' and its parents during fruit development
}

\section{Xiaojie Liu}

Northwest Agriculture and Forestry University

Nini Hao

Northwest Agriculture and Forestry University

\section{Ruifang Feng}

Northwest Agriculture and Forestry University

\section{Zhipeng Meng}

Northwest Agriculture and Forestry University

\section{Yanan Li}

Northwest Agriculture and Forestry University zhengyang zhao ( $\sim$ zhaozy@nwsuaf.edu.cn ) Northwest Agriculture and Forestry University

\section{Research article}

Keywords: Volatile aroma compounds, 'Ruixue' (Malus $\times$ domestica Borkh.), Fruit flavour, gas chromatography-mass spectrometry (GC-MS), Gene expression

Posted Date: December 8th, 2020

DOl: https://doi.org/10.21203/rs.3.rs-51327/v2

License: (c) (i) This work is licensed under a Creative Commons Attribution 4.0 International License. Read Full License 


\section{Abstract}

Background: Aroma is one the most crucial inherent quality attributes of fruit. 'Ruixue' apples were selected from a cross between 'Pink Lady' and 'Fuji', a later ripening yellow new cultivar. However, there is little known about the content and composition of aroma compounds in 'Ruixue' apples or the genetic characters of 'Ruixue' and its parents. In addition, the metabolic pathways for biosynthesis of aroma volatiles and aroma-related genes remain poorly understood.

Results: Volatile aroma compounds were identified using gas chromatography-mass spectrometry (GCMS). Our results show that the aroma profile of volatile compounds changes with ripening. Aldehydes were the dominant volatile compounds in early fruit development, with alcohols and esters increasing dramatically during maturation. In ripe fruit, esters and terpenoids were the main aroma volatiles in ripening fruit of 'Pink Lady' and 'Fuji' apples, and they included butyl 2-methylbutanoate, propanoic acid, hexyl ester, propanoic acid, hexyl ester, hexanoic acid, hexyl ester, acetic acid, hexyl ester and (Z, E)-afarnesene. Interestingly, aldehydes and terpenoids were the dominant volatile aroma compounds in ripening fruit of 'Ruixue', and they mainly included 2-hexenal, 2-hexenal, octanal, (E)-2-octenal, nonanal and $(\mathrm{Z}, \mathrm{E})$-a-farnesene. By comparing the transcriptome profiles of 'Ruixue' and its parents fruits during development, we identified a large number of aroma-related genes related to the fatty acid, isoleucine and sesquiterpenoid metabolism pathways and transcription factors that may regulate aroma biosynthesis.

Conclusions: Our initial study facilitates a better understanding of the volatile aroma compounds that affect fruit flavour as well as the mechanisms underlying differences in flavour between 'Ruixue' and its parents.

\section{Background}

The quality of fruit is mainly determined by appearance, texture, flavour and nutritional properties [1]. Fruit flavour, one the most crucial inherent quality attributes, is determined by sugars, organic acids and aroma [2]. Fruit has a strong aroma that can directly reflect its flavour characteristics, a crucial factor affecting consumer acceptance and market competitiveness [3]. The approximately 2000 volatile compounds associated with aromas in fruits are highly complex and diverse. To date, approximately 350 volatile compounds have been identified and quantified and in apple [2], mainly identifiable as esters, alcohols, aldehydes, phenols, ethers, terpenes, ketones and some sulphur compounds. Among these only a small number contribute prominently to fruit aroma $[2,4]$.

Volatile aroma compounds are produced through four primary metabolic pathways including the fatty acid, isoleucine, mevalonate and phenylpropanoid pathways [5]. Aldehydes, alcohols and esters (straightchain) are synthesized from fatty acid metabolic pathways through $\beta$-oxidation and lipoxygenase activity (LOX). The lipoxygenase (LOX) pathway involves lipoxygenase (LOX), hydroperoxidelyase (HPL), alcohol dehydrogenase (ADH) and alcohol acyl transferases (AAT) [6]. Lipoxygenase (LOX) is the initial step in ester (straight-chain) biosynthesis during fatty acid degradation (linoleic and linolenic). In apples a total 
of 23 potential functional lipoxygenase (LOX) genes have been identified. Gene expression analysis and QTL mapping experiments indicate that MdLOX1a and MdLOX5e are involved in fruit aroma volatile production in a molecular breeding approach [7]. Alcohol acyl transferases (AAT) are involved in the final steps and in rate limiting ester biosynthesis [8]. Alcohol acyl transferase 1 (MPAAT1) has been isolated from 'Royal Gala' apples, catalysing the synthesis of the primary esters [9]. MpAAT1 has been co-located with ester QTLs (LG2) and transgenic apples (AAT1 knockdown lines) exhibiting a significant decrease in total ester concentrations, especially in 2-methylbutyl acetate and butyl propanoate [10]. In addition, alcohol acyl transferase 2 (MdAAT2) is highly expressed and significantly correlated with ester production in 'Granny Smith' apples [11]. It is reported that there is differential expression of AAT gene family members in different apple cultivars [12]. Distinct plant genotype and substrate specificity of AAT enzymes is a prominent factor contributing to cultivar differences in production of ester volatiles $[6,13]$. Terpenes are produced by the mevalonate (MVA) pathway in the cytoplasm [5]. Citrus contains abundant terpenoids such as $d$-limonene, $\beta$-pinene, $\beta$-linalool and valencene [14]. Terpene synthases (TPS) are the key enzymes in synthesis of volatile terpenes. In apple, a total of 55 putative apple TPS family members were identified, and functional analysis found that only 10 TPS genes are predicted to be functional, synthesizing most of the terpene volatiles produced in 'Royal Gala' apples such as D-germacrene, linalool and a-pinene [15]. It is worth noting that a-farnesene synthase (MdAFS1) of TPS enzymes and its oxidative product have been identified as key factors causing superficial scald, a postharvest physiological disorder [16].

The differences in content and composition of aroma compounds among varieties produce different flavours in fruit. In 'Golden Delicious' apples a total of 36 volatile aroma compounds have been identified and quantified. Among these, esters accounted for greater than $80 \%$ of total volatiles, and butylacetate and hexylacetate were the primary compounds [17]. In 'Fuji' apples, a total of 30 volatile aroma compounds have been identified and quantified. Among these, esters and alcohol have been identified as dominant, accounting for $70 \%$ of total aroma, with 2-methylbutyl acetate, butyl acetate, hexyl acetate, and 2-methyl-1-butanol the most abundant [8]. In 'Pink Lady' apples, 31 volatile compounds were detected, with esters representing approximately $98 \%$ of the total. The main volatile compounds were hexyl acetate followed by hexyl hexanoate, hexyl 2-methylbutanoate, hexyl butanoate and hexyl propanoate [18]. Recently, the characteristic aroma of 'Honeycrisp' apples was identified and quantified using gas chromatography-olfactometry (GC-0) and aroma extract dilution analysis (AEDA) [4]. Hexylmethylbutyrate, hexyl 2-methylbutyrate, a-farnesene, and (E)-2-hexenal were dominant contributors to the aroma of 'Honeycrisp' apples.

'Ruixue' apples were selected from a cross between 'Pink Lady' and 'Fuji', a later ripening yellow apple cultivar. A preliminary study of the quality of 'Ruixue' fruit in our laboratory, sensory analysis shows that its flesh is crisp囚juicy, and sweet囚having a distinct aromatic flavour compared to its parents [19]. However, there is little known about the content and composition of aroma compounds in 'Ruixue' apples or the genetic characters of 'Ruixue' and its parents. In addition, the metabolic pathways for biosynthesis of aroma volatiles and aroma-related genes remain poorly understood. In this study, volatile aroma compounds were identified and quantified for 'Ruixue', 'Pink Lady' and 'Fuji' apples during fruit 
development using gas chromatography-mass spectrometry (GC-MS) and compared between the three cultivars. RNA sequencing (RNA-seq) transcriptome data and quantitative reverse transcriptase-PCR were used to identify candidate structural genes and potential transactional factors associated with aroma synthesis by the fatty acid, isoleucine and sesquiterpenoid metabolism pathways in 'Ruixue', 'Pink Lady' and 'Fuji' apples during fruit development. This study aims to clarify aroma profiles and further reveal the mechanisms underlying differential volatile aroma compounds between 'Ruixue' and its parents that affect the flavour of these three cultivars.

\section{Results}

\section{Physiological characteristics of 'Ruixue' and its parents apples during fruit development}

The 'Ruixue' apple, a yellow cultivar, was selected from a cross between 'Pink Lady' and 'Fuji' (Fig.1A). A total of six developmental stages were collected from 120 DAFB to the ripened stages. The colour of the 'Ruixue' apple peel changed from green to yellow and the colour of the pulp from green to milky white (Fig.1B). Fruit firmness, total soluble solids and titrable acidity are critical factors for fruit quality. Fruit firmness of the three cultivars showed a significant decline before 150 DAFB (Fig.1C), and then declined continually during latter development. There were no significant differences in fruit firmness between 'Ruixue' and 'Pink Lady' at 200 DAFB. However, the fruit firmness of 'Ruixue' was greater, approximately 1.3-fold that of 'Fuji'. The total soluble solids of the three cultivars showed a significant increase during fruit development. The total soluble solid content of 'Ruixue' was $17.6 \%$, greater than those of 'Pink Lady' and 'Fuji' at 200 DAFB (Fig.1D). The titrable acidity of the three cultivars showed a first increase, and then slowly declined during fruit development (Fig.1D). The titrable acidity of 'Ruixue' was far less than that of

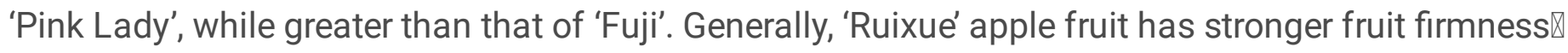
higher sugar and lower acidity.

\section{Aroma volatiles profile of 'Ruixue' and its parents apples during fruit development}

Fruit aroma is a crucial quality attribute, playing a vital role in consumer selection and acceptance. The volatile aroma compounds and relative changes in content of 'Ruixue' and its parents apples were identified during different fruit developmental stages. A total of 54 aroma volatiles were monitored from 'Ruixue' $\nabla^{\prime} F u j i$ ' and 'Pink Lady' during fruit development (Supplementary Table S1), including 35 esters (the most abundant volatiles), 9 aldehydes, 5 alcohols, 4 terpenoids and 1 acid. 'Ruixue' contained 40 aroma volatiles: 25 esters, 8 aldehydes, 3 alcohols, 3 terpenoids and 1 acid. 'Fuji' contained 46 aroma volatiles: 29 esters, 9 aldehydes, 4 alcohols, 3 terpenoids and 1 acid. 'Pink Lady' contained only 32 aroma volatiles: 22 esters, 4 aldehydes, 3 alcohols, 2 terpenoids and 1 acid.

The total aroma volatile contents of 'Ruixue' and its parents apples increased slowly from 120 DAFB to 150 DAFB then increased rapidly from 150 DAFB to 200 DAFB. In contrast to 'Fuji' (190 DAFB), the total aroma volatiles content of 'Ruixue' and 'Pink Lady' reached maximum levels at 200 DAFB (Fig.2A). The relative content of aroma volatiles (esters, aldehydes, alcohols, terpenoids and acids) during fruit development exhibited dynamic change (Fig.2B, C and D). For 'Ruixue', aldehydes were the main aroma 
volatiles and account for more than $96 \%$ of total aroma volatiles prior to 170 DFAB (immature fruit), then declined to a minimum $₫ 32.19 \%$ Wat 200 DAFB (mature fruit). Esters began to accumulate after $170 \mathrm{DAFB}$, reaching a maximum (29.91\%) at 200 DAFB. Similarly the relative terpenoid content exhibited a significant increase after 170 DAFB, reaching its maximum (32.91\%) at $200 \mathrm{DAFB}$, becoming the most prominent aroma volatiles. Few alcohols and acids were detected during fruit development, and the results for 'Pink Lady' and 'Fuji' were similar. For 'Pink Lady', aldehydes were the predominant aroma volatiles and account for more than $90 \%$ of total aroma volatile content before 170 DFAB (immature fruit) then rapidly decreased to a minimum $₫ 4.28 \% \bigotimes$ at 200 DAFB. Conversely, terpenoids began to accumulate after 170 DAFB, reaching a maximum (53.37\%) at 200 DAFB. For 'Fuji', the relative content of aldehydes continued to decrease during fruit development and reached a minimum (14.95\%) at 200 DAFB; however, the relative content of esters increased to a maximum level (63.92\%) at 190 DAFB and then declined.

To visualise the apple fruit aroma volatiles profile of the maturation stage, aroma volatiles of 'Ruixue' $\rrbracket^{\prime} F u j i$ ' and 'Pink Lady' at 200 DAFB were analysed for hierarchical clustering (Fig. 2E). A total of 29 volatiles were detected in 'Ruixue' apple fruit, including 17 esters, 6 aldehydes, 2 alcohols, 3 terpenoids and 1 acid. For 'Pink Lady' a total of 28 aroma volatiles were identified, including 21 esters, 3 aldehydes, 1 alcohol, 2 terpenoids and 1 acid. In 'Fuji' apples, 31 aroma volatiles were identified, including 22 esters, 5 aldehydes, 2 alcohols, 1 terpenoid and 1 acid. Interestingly, in 'Ruixue' apples, aldehydes (2-hexenal, 2hexenal and (E)-2-octenal), esters (butanoic acid, propyl ester, butanoic acid, 2-methylbutyl ester,

propanoic acid, 2-methyl-, pentyl ester), alcohols (alcohol) and terpenoids (5-hepten-2-one, 6-methyl-) were significantly higher than in 'Fuji' and 'Pink Lady'. Conversely, esters (butyl 2-methylbutanoate, propanoic acid, hexyl ester, butanoic acid, butyl ester, heptanoic acid, butyl ester, hexanoic acid, butyl ester and hexanoic acid, and hexyl ester) were significantly lower than in 'Fuji' and 'Pink Lady'. In summary, there were differences in aroma volatile composition and content in different apple cultivars.

\section{Transcriptome analysis of 'Ruixue' and its parents apples during fruit development}

In order to further explore the molecular mechanisms underlying differences in flavour between 'Ruixue' and its parents, RNA-sequencing (RNA-Seq) was utilized to obtain genome-wide gene expression profiles during fruit development with same samples used for volatile compound analysis. A total of 54 samples (three cultivars $\times$ six developmental stages $\times$ three biological replicates) were subjected to RNA-seq analysis in order to identify differentially expressed genes related to volatile compound biosynthesis and transcriptional regulation responsible for the diverse flavours exhibited by the three cultivars. After filtering, a total of 1073298706 clean reads were obtained from 'Ruixue'fruit, 979721018 and 1015631116 clean reads were produced from'Fuji' and 'Pink Lady' fruit, respectively. A total of $460.76 \mathrm{~GB}$ nucleotides were obtained with an average GC content of 47.28\%. Q30 percentage (error rates lower than $0.3 \%$ ) was over $90 \%$ (Supplementary Table S2).

Principal component analysis was based on the transcriptome profiles from 18 samples. The first two principal components explain $41.63 \%$ (PC1) and $21.20 \%$ (PC2) of the variance among the samples. Similarities and differences among the apple transcriptomes were mostly driven by fruit developmental 
stage. Additionally, 'Ruixue'囚 'Fuji' and 'Pink Lady' samples were separated by PC1 (Fig. 3A). The number of differentially expressed genes (DEGs) showed first an increase and then a decrease during fruit development (Fig. 3B). The number of DEGs was most abundant in the middle and later stages of development (150-170 DAFB).

KEGG pathway analysis was used to ascertain the potential involvement of metabolic pathways in the regulation of fruit aroma volatile synthesis. Since the majority of volatile aroma compounds were synthesized, and the numbers of DEGs most abundant, in the middle and later stages of fruit development, we selected comparisons of X3 versus F3 and X3 versus P3 ('Ruixue', 'Fuji' and 'Pink Lady' fruit at 170 DAFB) for KEGG pathway analysis (Supplementary Table 1; Fig. 3B). The DEGs mapped to 110 KEGG pathways in the pairwise comparison of X3 versus $F 3$, with the greatest number of DEGs mapped to phenylpropanoid biosynthesis (mdm00940) (Fig. 3C). Volatile aroma compound synthesis pathways included sesquiterpenoid and triterpenoid biosynthesis, and fatty acid degradation was also significantly enriched in KEGG. A comparison (X3 versus P3囚of DEGs resulted in the identification of 114 KEGG pathways, with significant enriched pathways identified as plant-pathogen interaction (mdm04626), phenylpropanoid biosynthesis (mdm00940) and sesquiterpenoid and triterpenoid biosynthesis (mdm00909) (Fig. 3D). In general, the KEGG enrichment analyses indicated that fatty acid metabolism, phenylpropanoid metabolism and sesquiterpenoid metabolism play a critical role in fruit aroma volatile synthesis in 'Ruixue', 'Fuji' and 'Pink Lady'.

\section{Expression changes in aroma-related genes involved in fatty acid, isoleucine and sesquiterpenoid metabolism pathways}

Esters (straight and branched chain esters) are among the most abundant volatile aroma compounds in apple fruits. Straight chain esters are produced by the fatty acid metabolism pathway while branched chain esters are produced by the isoleucine metabolism pathway (Fig. 4A). The expression levels of numerous genes showed significant increases, but were differently expressed in the different cultivars during fruit development (Fig. 4B). DH-1(3-hydroxyacyl ACP dehydratase) exhibited a very low expression level in 'Ruixue', significantly lower than in 'Fuji' during fruit development. Interestingly, ER-1 and ER-2 (2, 3-trans-enoyl ACP reductase) had an opposite expression pattern. Lipoxygenases (LOX) are involved in the initial steps of ester biosynthesis in fatty acid degradation, including six genes (LOX-1 to LOX-6). Four lipoxygenases (LOX-1 to LOX-4) dramatically increased during fruit development, transcribed at high levels in 'Ruixue' but at low levels in 'Pink Lady'. However, LOX-5 and LOX-6 showed high expression levels prior to 170 DAFB then decreased rapidly. Hydroperoxidelyase (HPL) is the final step toward biosynthesis of aldehydes (hexanal) with an expression level that first increased then decreased. Alcohol dehydrogenase reduced aldehydes from the fatty acid and isoleucine degradation pathways to alcohols. Transcript abundance of ADH-1, ADH-2 and ADH-3 continuously deceased during fruit development. Alcohol acyl transferases (AAT) are the rate limiting enzymes for ester biosynthesis. Transcript abundance of AAT-1, AAT-2 and AAT-3 continuously increased during fruit development. It is noteworthy that the transcript abundance of AAT in 'Ruixue' was lower than in 'Fuji' and 'Pink Lady'. Aldehyde dehydrogenase (ALDH-1 to ALDH-3) and carboxylesterase (CXE-1 to CXE-2) are important enzymes in 
biosynthesis of branched chain esters by the isoleucine metabolism pathway and were differentially expressed during fruit development.

Sesquiterpenes are the most prominent terpenoids in apples. The main sesquiterpene that accumulated was (Z, E)-a-farnesene, the most abundant of any aroma volatile compounds (Supplementary Table S1). Sesquiterpenes are produced by the mevalonate (MVA) pathway in the cytoplasm in nine enzymatic steps (Fig. 5A). Expression levels of numerous genes showed significant increases during fruit development. There were different expressions of sesquiterpene biosynthetic genes in the different cultivars during fruit development. Notably, the expression levels of a-farnesene synthase (AFS) exhibited drastic increases, higher in 'Pink Lady' than in 'Fuji' and 'Ruixue'. Similarly, a-Farnesene exhibited rapid increases during fruit development with the same expression pattern as AFS (Fig. 5 B).

\section{Expression changes in potential transcriptional factors}

Transcriptional factors play a crucial role in regulating aroma volatile synthesis. To clarify potential transcription factors that may be involved, we further analysed expression patterns of transcription factors during fruit development, including MYC2, ERF, WRKY, MYB, BZIP and MADS-box TFs. The expression patterns of numerous transcription factors increased during fruit development, but were differently expressed in the different cultivars (Supplementary Fig. S1).

\section{Quantitative reverse transcriptase-PCR validation of the transcriptome data}

To validate the reliability and repeatability of the transcriptome data, 6 aroma biosynthesis and 3 transcription factor genes were selected for analysis of their expression levels using qRT-PCR. Genespecific primers used in this analysis are listed in Supplementary Table S3. The expression profiles of 9 candidate genes generated using qPCR were very similar to the RNA-Seq results (RPKM values), which had high a Pearson correlation coefficient $\left(R^{2}>0.9\right)$. These results indicate that transcriptomic data were accurate and reproducible.

\section{Discussion}

Dynamic variations of volatile compounds in the aroma profiles of 'Ruixue' and its parents apples during fruit development

Aroma is a crucial indicator of fruit flavour that has been used to distinguish varieties of fruit and fruit cultivars [3]. Aroma profiles of apple volatile compounds are extremely complex, with aroma composition and concentration influenced by cultivar-specific traits and fruit development stages [5]. The aroma profiles of volatile compounds change with ripening. Aldehydes are dominant volatile compounds in the early stages of fruit development [20], but their content decreases with fruit ripening followed by dramatic increases in alcohols, and finally esters are dominant at fruit maturation [2]. 
The volatile aroma compounds of 'Ruixue', 'Pink Lady' and 'Fuji' apples were determined during fruit development. A total of 40, 46 and 32 volatile compounds were detected in 'Ruixue', 'Pink Lady' and 'Fuji' apples, respectively, including esters, aldehydes, alcohols, terpenoids and acids. Aldehydes were the predominant aroma volatiles and account for more than $90 \%$ of total aroma volatiles in the three cultivars during the early stages of fruit development. Alcohols and esters showed a significant increase during the latter stages of fruit development in all cultivars. It has been shown that esters are the most important contributors to fruit flavour, accounting for more than $80 \%$ of the total volatile compounds in 'Granny Smith' [21], 'Gala' and 'Starking Delicious' apples [22-23]. Similar studies show that esters are important volatile aroma compounds that significantly affect fruit flavour in bananas and strawberries [24-26]. Our data show that esters and terpenoids were the main aroma volatiles in ripening fruit of 'Pink Lady' and 'Fuji' apples, mainly including butyl 2-methylbutanoate, propanoic acid, hexyl ester, propanoic acid, hexyl ester囚hexanoic acid, hexyl ester and acetic acid, hexyl ester and (Z, E)-a-farnesene. Among these, acetic acid, hexyl ester was identified as a characteristic aroma volatile compound, responsible for fruity notes [27]; however, acetic acid, hexyl ester was not detected in 'Ruixue' apples. Interestingly, aldehydes were the dominant volatile aroma compounds in ripening fruit of 'Ruixue', mainly including 2hexenal, 2-hexenal, octanal, (E)-2-octenal and nonanal, providing grassy and green notes [18]. In addition, 1-butanol, 2-methyl-in 'Ruixue' was significantly higher than in 'Pink Lady' and 'Fuji' apples, contributing to a sweetish sensation. Altogether, these results show that aldehyde-type flavours predominate in 'Ruixue', while 'Pink Lady' and 'Fuji' exhibit ester-type flavours. Differences in the aroma profiles of the 'Ruixue' cultivar and its parents and significant variations at different stages of 'Ruixue' fruit development result in a great diversity of flavours. In addition, our result show that 'Ruixue' apple fruit has stronger fruit firmness $₫$ higher sugar and lower acidity. Sweetness of fruit is one of the primary drivers of consumer preference. It has been shown apple sweetness is provided by some aroma compounds, mainly esters and farnesene. Taken together, the sugar, acid and aroma together determine the flavour of the fruit

\section{Analysis of expression patterns of aroma-related genes during fruit development involved in fatty acid, isoleucine and sesquiterpenoid metabolism pathways}

Previous studies have shown that numbers of volatile aroma compounds are synthesized mainly by the fatty acid, isoleucine, mevalonate and phenylpropanoid metabolism pathways [5]. Among these, fatty acid and isoleucine metabolism are the most important pathways for synthesis of aldehydes, alcohols and esters, associated with $\beta$-oxidation and lipoxygenase activity (LOX). The lipoxygenase pathway mainly comprises four crucial enzymes including lipoxygenase (LOX), hydroperoxidelyase (HPL), alcohol dehydrogenase (ADH) and alcohol acyl transferase (AAT) [6]. It has been shown that lipoxygenases (LOX) are involved in the initial steps toward biosynthesis of aldehydes, alcohols and esters involved in fatty acid degradation, with linoleic and linolenic being two important substrates for LOX [28]. 23, 11 putative LOX genes were identified in Golden Delicious and Royal Gala apples, respectively [5, 7]. Recent studies show that MdLOX1a and MdLOX5e are involved in fruit aroma volatile production, arguing for a molecular breeding approach [7]. In our study, six differentially expressed genes (LOX-1 to LOX-6) were identified in 'Ruixue', 'Fuji' and 'Pink Lady'. Among these, four lipoxygenases (LOX-1 to LOX-4) dramatically increased during fruit development, transcribed at high levels in 'Ruixue' but at low levels in 
'Pink Lady'. These observations are in agreement with increases in aldehydes during fruit development and aldehyde concentrations in 'Ruixue' fruit greater than those in 'Pink Lady', suggesting that LOX-1, LOX-2, LOX-3 and LOX-4 play a key positive role in the biosynthesis of aldehydes. Similar results were reported previously in Korla fragrant pears (Pyrus sinkiangensis Yu) [29]. However, LOX-5 and LOX-6 first increased and then rapidly decreased. Alcohol dehydrogenase (ADH) reduced aldehydes from the fatty acid and isoleucine degradation pathways to alcohols [2]. It has been shown that ADH activity decreased in 'Fuji' apples during fruit development [21]. Conversely, ADH activity increased until harvest in 'Jonagold' apple [30]. In addition, transcript levels of ADH decreased during fruit development [29]. Here we have identified three $A D H$ genes and our results are similar to these previous studies in that transcript abundances of $A D H-1, A D H-2$ and $A D H-3$ continuously deceased during fruit development. However, content of the alcohols compounds showed an increasing trend during fruit development. These results indicate that regulation of $\mathrm{ADH}$ occurs at the post-transcriptional and translational level [8]. Alcohol acyl transferases (AAT) are the rate limiting enzymes of ester biosynthesis. Studies have shown that AAT activity increases with fruit ripening in 'Granny Smith' and 'Fuji' apples [13]. Transcript levels of AAT1-GS and $A A T 1-R G$ increased during fruit development in 'Granny Smith' and 'Royal Gala' apples [31]. Our data also show that transcript abundances of AAT-1, AAT-2 and AAT-3 continuously increase during fruit development. It is also notable that the transcript abundance of AAT in 'Ruixue' was lower than in 'Fuji' and 'Pink Lady'. This is consistent with the observation that the ester content of 'Ruixue' is lower than in 'Fuji' and 'Pink Lady'. Terpenes are produced from the mevalonate (MVA) pathway in the cytoplasm [5]. Terpene synthases (TPS) are the key enzymes for synthesising terpene volatiles. To date, 55 putative apple TPSs have been identified and only 10 TPS genes are predicted to be functional, able to synthesize most of the terpene volatiles produced in the 'Royal Gala' such as D-germacrene, linalool and a-pinene. However, we detected only the terpene volatile a-farnesene (sesquiterpenes), synthesized by a-farnesene synthase (MdAFS1). Expression levels of a-farnesene synthase (AFS) drastically increase during fruit development and were higher in 'Pink Lady' than in 'Fuji' and 'Ruixue'. Differentially expressed aromarelated genes may be responsible for changes in 'Ruixue' and its parents apples aroma compounds. Altogether, these results show that aroma synthesis is a complex and precise process that involves multiple structural gene families and metabolic pathways.

\section{Expression changes in candidate transcription factors that may regulate aroma biosynthesis}

Previous studies have shown that transcription factors (TFs) play a primary role in regulating volatile plant secondary metabolites [32]. To further study transcription factors that may regulate aroma biosynthesis, We analysed the expression patterns of transcription factors that may be involved in the regulation of aroma synthesis. It has been reported that $A t M Y C 2$, a bHLH transcription factor, promotes sesquiterpene production by binding to promoters of TPS21 and TPS11 in Arabidopsis thaliana flowers then activates their transcription, mediating both GA and JA signals [32]. Here『we have identified two MYC2 genes (MYC2-1 and MYC2-2). Transcript levels of MYC2-1 were high during early and late stages of fruit development. This indicates that MYC2-1 may be involved in regulating aldehyde synthesis during the early stages and ester synthesis during the late stages of fruit development. However, transcript abundances of MYC2-2 maintained a lower level during fruit development. Research has shown that 
ERF1 and ERF2 of AP2/ERF transcription factors are involved in regulating sesquiterpene synthase amorpha-4,11-diene synthase[33]. In oranges, CitAP2.10 promotes (+)-valencene content via activation of the terpene synthase CsTPS1 [14]. Recently, an ethylene response factor (FaERF\#9) has been shown to activate FaQR transcripts and to up-regulate furanone biosynthesis in strawberries [34]. These results indicate that ERF predominantly regulates terpene biosynthesis. Our results show that transcript abundances of ERF-1, ERF-1, ERF-3 and ERF-4 increased during fruit development; however, the transcript abundance of ERF-2 first increased then decreased. In accordance with previous studies and our data, ERF may be involved in regulating ( $Z, E$ )-a-farnesene biosynthesis in apples. It has been reported that GaWRKY1, WRKY transcription factors regulate sesquiterpene biosynthesis in cotton via activation of the (+) - $\delta$-cadinene synthase (CAD1) transcript [35]. In addition, the MYB transcription factor (FaMYB98) upregulates furanone biosynthesis in strawberries [34]. Therefore, further functional studies will be required to clarify the regulatory roles of these transcription factors in apple aroma biosynthesis.

\section{Conclusions}

The aroma volatiles profile of 'Ruixue' and its parents apples during fruit development were identified using SPME-GC-MS. Our results show that the aroma profile of volatile compounds changes with ripening. Aldehydes were dominant volatile compounds during the early stages of fruit development. In ripe fruit, esters and terpenoids are the main aroma volatiles in ripening fruit of 'Pink Lady' and 'Fuji' apples, and they mainly included butyl 2-methylbutanoate, propanoic acid, hexyl ester, propanoic acid, hexyl ester, hexanoic acid, hexyl ester, acetic acid, hexyl ester and (Z, E)-a-farnesene. Interestingly, aldehydes and terpenoids were dominant volatile aroma compounds in ripening fruit of 'Ruixue', mainly 2hexenal, 2-hexenal, octanal, (E)-2-octenal, nonanal and (Z, E)-a-farnesene. By comparing the transcriptome profiles of 'Ruixue' and its parents during development. We identified a large number of aroma-related genes involved in the fatty acid, isoleucine and sesquiterpenoid metabolism pathways and identified transcription factors that may regulate aroma biosynthesis. Our initial study facilitates a better understanding of the volatile aroma compounds that affect fruit flavour as well as the mechanisms underlying differences in flavour between 'Ruixue' and its parents.

\section{Methods}

\section{Plant material}

Fruit of three apple cultivars, new cultivars 'Ruixue' (An application will be made to protect 'Ruixue' nationally in China. Trees and bud wood under rules of international Plant Variety Rights are available for research purposes, which can be obtained from Zhengyang Zhao at the College of Horticulture, Northwest A\&F University, Yangling, Shaanxi, China. The number of cultivar: CNA20151469.1), 'Pink Lady' and 'Fuji', were collected from the national apple model of Northwest A\&F University in Baishui County, Weinan City, Shaanxi Province, China. The three apple cultivars (6-year-old) were grafted onto M26 rootstock and planted in $4.0 \times 1.5 \mathrm{~m}$ plots. Fruit of uniform appearance, without mechanical damage and disease were collected from 'Ruixue', 'Pink Lady' and 'Fuji' at six different developmental stages, 120, 150, 170, 180, 
190 and 200 days after full bloom (DAFB). Three independent replicates were performed for each sample, consisting of 10 fruits each from 15 apple trees. A total of 18 fruit samples (three cultivars $\mathrm{x}$ six developmental stages $\times$ three biological replicates) were frozen in liquid nitrogen then stored at $-80^{\circ} \mathrm{C}$ for GC-MS and RNA-Seq analysis.

\section{Mensuration of fruit physiological characteristics}

Firmness of flesh was measured using a GS-15 fruit texture analyser (Guss Manufacturing Pty. Ltd., Cape Town, South Africa) on both sides of the fruit equator of peeled fruits (test depth, $8 \mathrm{~mm}$; diameter probe,10 mm). Total soluble solids (TSS) were measured using a hand-held refractometer (Pocket PAL-1, ATAGO, Tokyo, Japan). Titrable acidity (TA) was measured using a digital fruit acidity meter (GMK-835F Perfect, Germany). All samples were measured for each cultivar ('Ruixue', 'Pink Lady' and 'Fuji') at each time point $(120,150,170,180,190$ and 200 DAFB).

\section{Analysis of volatile aroma compounds using gas chromatography-mass spectrometry (GC-MS)}

Solid phase micro extraction (SPME) was used to extract the volatile components of fruit with three biological replicates for each sample [36]. Approximately $5.0 \mathrm{~g}$ of fruit frozen to $-80^{\circ} \mathrm{C}$ was ground into powder and sealed in a $50 \mathrm{~mL}$ vial, adding $1.0 \mathrm{~g} \mathrm{NaCl}$ and $10 \mu \mathrm{L}$ 3-nonanone $\nabla 0.04 \mu \mathrm{L} \mathrm{mL}{ }^{-1} \rrbracket a s$ an internal standard, then the headspace gases were extracted for 40 min at $40^{\circ} \mathrm{C}$ using fibre coated with $50 / 30 \mu \mathrm{m}$ divinylbenzene/carboxen/polydimethylsiloxane (DVB/CAR/PDMS). Subsequently, samples were directly injected into the injection port of a GC-MS (Thermo Fisher Scientific Inc., USA, ISQ, TRACE GC ULTRA) with a splitless mode for $2.5 \mathrm{~min}$ at $230^{\circ} \mathrm{C}$. Volatile analysis was performed using a HP-INNO wax column (60 $\mathrm{m} \times 0.25 \mathrm{~mm}$ inner diameter $\times 0.25 \mu \mathrm{m}$ film thickness) with a carrier gas helium flow rate of $34 \mathrm{~cm} \mathrm{~s}$

1 . The initial oven temperature was maintained at $40^{\circ} \mathrm{C}$ for $3 \mathrm{~min}$, followed by a temperature increase at a rate of $5^{\circ} \mathrm{C} \mathrm{min}-1$ to $150{ }^{\circ} \mathrm{C}$, then to $220^{\circ} \mathrm{C}$ at a rate of $10{ }^{\circ} \mathrm{C} \mathrm{min}-1$ then maintained at $220^{\circ} \mathrm{C} \mathrm{for} 5 \mathrm{~min}$. The energy of electron impact (EI) was $70 \mathrm{eV}$. The transfer temperature and source temperature were 240 ${ }^{\circ} \mathrm{C}$. Aroma volatiles were qualitatively analysed by comparison with mass spectra (MS) from the National Institute of Standards and Technology (NIST) and Mass Spectral Library (NIST2014). Aroma volatiles were quantitatively analysed using the peak area of the internal standard (3-nonanone) as a reference based on the total ion chromatogram (TIC).

\section{RNA extraction and RNA sequencing analysis}

A total of 54 samples (three cultivars $\times$ six developmental stages $\times$ three biological replicates) were subjected to transcriptome analyses. Total RNA was extracted from fruit of 'Ruixue', 'Pink Lady' and 'Fuji' at 120, 150, 170, 180, 190 and 200 DAFB using the CTAB method [37]. RNA integrity was determined using $1.2 \%$ agarose gel electrophoresis. Quantitation of RNA was analysed using a NanoDrop ND1000 spectrophotometer (NanoDrop Technologies, Wilmington, DE, USA) and RNA quality was analysed with an Agilent Bioanalyzer 2100 system (Agilent Technologies, Palo Alto, CA, USA). The agarose gel for RNA integrity was fine. A260/A280 values are between 1.8-2.0, A260/A230 value is more than 2.0. mRNA (containing polA) was isolated from total RNA using magnetic beads with oligo (dT). Fifty-four cDNA 
libraries were constructed using the NEBNext ${ }^{\circledR}$ UltraTM RNA Library Prep Kit for Illumina ${ }^{\circledR}$ (NEB, USA) and sequenced on the Illumina HiSeq platform. To ensure the quality and reliability of transcriptome data analysis, low-quality reads and N-containing reads were removed from the raw RNA-seq reads.

Transcript abundance was quantified using HISAT2 software and normalized using the fragments-perkilobase-of-transcript-per-million-mapped-reads (FPKM) method. Differentially expressed genes (DEGs) were identified using DESeq2 with log2 (fold change) $\geq 1$ and corrected $P \leq 0.005$. All identified DEGs were subjected to gene-ontology (GO) enrichment and KEGG pathway analysis with padj $\leq 0.05$ [38-39].

\section{Quantitative reverse transcriptase-PCR validation of the transcriptome data}

Total RNA was extracted using the CTAB method [23]. The extracted RNA was synthesized into firststrand cDNA from $1 \mu \mathrm{g}$ of total RNA using a PrimeScript RT reagent kit with gDNA Eraser (Takara Bio, Shiga, Japan) according to the manufacturer's instructions. qPCR was performed using a StepOneplus ${ }^{T M}$ instrument (Thermo Fisher Scientific Inc., USA). Real-time PCR reaction volumes of $20 \mu \mathrm{L}$ included $10 \mu \mathrm{L}$ $2 \times$ SYBR Green PCR Master Mix (Vazyme), $2 \mu \mathrm{L}$ of cDNA, $0.8 \mu \mathrm{L}$ of each primer, $0.4 \mu \mathrm{L} 50 \times \mathrm{ROX}$ Reference and $6.8 \mu \mathrm{L}$ distilled deionized $\mathrm{H}_{2} \mathrm{O}$. Gene-specific primers used in the qRT-PCR analysis are listed in Supplementary Table S4. The qPCR protocol included initial denaturation at $95^{\circ} \mathrm{C}$ for $5 \mathrm{~min}$, followed by 40 cycles of $95^{\circ} \mathrm{C}$ for $15 \mathrm{sec}$ and $60^{\circ} \mathrm{C}$ for $30 \mathrm{sec}$. Melting-curve analysis was used to verify gene-specific PCR products (with an initial step of $94^{\circ} \mathrm{C}$ for $15 \mathrm{sec}$, second step of $60^{\circ} \mathrm{C}$ for $60 \mathrm{sec}$, finally $95^{\circ} \mathrm{C}$ for $15 \mathrm{sec}$ ). An apple actin gene was used as a reference gene to normalize all gene expression levels. The $2^{-\Delta \Delta C t}$ method was used to calculate relative expression levels of genes [40].

\section{Statistical Analysis}

Figures were drawn using ORIGIN 2019 (Microcal Software, Inc., Northampton, MA). Significant difference analyses $(P<0.05)$ were carried out using one-way ANOVA with Duncan's test (SPSS 19.0, SPSS Inc., Chicago, IL). Gene ontology (GO) enrichment of the differential expressed genes (DEGs) was performed using the GOseq R packages[41]. Kyoto Encyclopedia of Genes and Genomes (KEGG) enrichment analysis of the DEGs was implemented by the Bio-conductor package cluster Profiler version 3.10.14[42]

\section{Abbreviations}

mRNA: Messenger RNA; MYC2: Myelocytomatosis protein 2; bHLH: basic Helix-Loop-Helix; qRT-PCR: Quantitative reverse transcriptase polymerase chain reaction; CTAB: Cetyltrimethylammonium bromide; RNA: Ribonucleic Acid; cDNA: complementary Deoxyribonucleic Acid.

\section{Declarations}

\section{Acknowledgements}

Not applicable 


\section{Authors' contributions}

$X \mathrm{~L}$ and $\mathrm{ZZ}$ Z designed this study; $\mathrm{X} L$ wrote the manuscript; $N \mathrm{H}, \mathrm{R} \mathrm{F}, \mathrm{Z} \mathrm{M}$ and $\mathrm{Y} \mathrm{L}$ performed the experiments and analysis. All authors have read and approved the manuscript.

\section{Funding}

This work was supported by the earmarked fund for the Special Project for Building Industrial Technology System of National Modern Agriculture (Apple) (CARS-28), the Modern Agro-industry Technology Research System, China (CARS-27); the Northwest A\&F University of Science and Technology (2014 YB 086). The funder has no role in the design of the study and collection, analysis, and interpretation of data and in writing the manuscript.

\section{Ethics declarations}

\section{Ethics approval and consent to participate}

Not applicable.

\section{Consent for publication}

Not applicable.

\section{Competing interests}

The authors declare that they have no competing interests.

\section{Availability of data and materials}

All data generated or analyzed during this study are included in this published article and its supplementary information files.

\section{References}

1. Song J, Forney CF. Flavour volatile production and regulation in fruit. Canadian Journal of Plant Science. 2008;88: 537-550.

2. Espinodiaz M, Sepulveda DR, Gonzalezaguilar GA, Olivas GI. Biochemistry of Apple Aroma: A Review. Food Technology and Biotechnology. 2016;54: 375-394.

3. Bonany J, Buehler A, Carbó J, Codarin S, Schoorl F. Consumer eating quality acceptance of new apple varieties in different European countries. Food Quality \& Preference. 2013;30: 250-259.

4. Yan D, Shi J, Ren X, et al. Insights into the aroma profiles and characteristic aroma of 'Honeycrisp' apple (Malus $x$ domestica). Food Chem. 2020;327: 127074. 
5. Schaffer RJ, Friel EN, Souleyre EJ, et al. A genomics approach reveals that aroma production in apple is controlled by ethylene predominantly at the final step in each biosynthetic pathway. Plant Physiol. 2007;144: 1899-1912.

6. Echeverría G, Graell J, López ML, Lara I. Volatile production, quality and aroma-related enzyme activities during maturation of 'Fuji' apples. Postharvest Biology and Technology. 2004;31: 217-227.

7. Vogt J, Schiller D, Ulrich D, Schwab W, Dunemann F. Identification of lipoxygenase (LOX) genes putatively involved in fruit flavour formation in apple (Malus $\times$ domestica). Tree Genetics \& Genomes. 2013;9: 1493-1511.

8. Defilippi BG, Kader AA, Dandekar AM. Apple aroma: alcohol acyltransferase, a rate limiting step for ester biosynthesis, is regulated by ethylene. Plant Science. 2005;168: 1199-1210.

9. Souleyre EJ, Greenwood DR, Friel EN, Karunairetnam S, Newcomb RD. An alcohol acyl transferase from apple (cv. Royal Gala), MpAAT1, produces esters involved in apple fruit flavor. FEBS J. 2005;272: 3132-3144.

10. Yauk YK, Souleyre EJF, Matich AJ, et al. Alcohol acyl transferase 1 links two distinct volatile pathways that produce esters and phenylpropenes in apple fruit. Plant J. 2017;91: 292-305.

11. Li D, Xu Y, Xu G, Gu L, Li D, Shu H. Molecular cloning and expression of a gene encoding alcohol acyltransferase (MdAAT2) from apple (cv. Golden Delicious). Phytochemistry. 2006;67: 658-667.

12. Zhu Y, Rudell DR, Mattheis JP. Characterization of cultivar differences in alcohol acyltransferase and 1-aminocyclopropane-1-carboxylate synthase gene expression and volatile ester emission during apple fruit maturation and ripening. Postharvest Biology and Technology. 2008;49: 330-339.

13. Holland D, Larkov O, Bar-Ya'akov I, et al. Developmental and Varietal Differences in Volatile Ester Formation and Acetyl-CoA: Alcohol Acetyl Transferase Activities in Apple (Malus domestica Borkh.) Fruit. Journal of agricultural and food chemistry. 2005;53: 7198-7203.

14. Shen SL, Yin XR, Zhang B, et al. CitAP2.10 activation of the terpene synthase CsTPS1 is associated with the synthesis of (+)-valencene in 'Newhall' orange. J Exp Bot. 2016;67: 4105-4115.

15. Nieuwenhuizen NJ, Green SA, Chen X, et al. Functional genomics reveals that a compact terpene synthase gene family can account for terpene volatile production in apple. Plant Physiol. 2013;161: 787-804.

16. Pechous SW, Whitaker BD. Cloning and functional expression of an (E, E)-alpha-farnesene synthase cDNA from peel tissue of apple fruit. Planta. 2004;219: 84-94.

17. Song J, Bangerth F. The effect of harvest date on aroma compound production from 'Golden Delicious' apple fruit and relationship to respiration and ethylene production. Postharvest Biology and Technology. 1996;8: 259-269.

18. López ML, Villatoro C, Fuentes T, Graell J, Lara I, Echeverría G. Volatile compounds, quality parameters and consumer acceptance of 'Pink Lady ${ }^{\circledR}$ ' apples stored in different conditions. Postharvest Biology and Technology. 2007;43: 55-66.

19. Li H, Liu J, Zhang X, et al. Comparison of textural and ultrastructural characteristics of four apple cultivars with different textures during cold storage. International Journal of Food Properties. 
2019;22: 659-669.

20. Mattheis JP, Fellman JK, Chen PM, Patterson ME. Changes in headspace volatiles during physiological development of Bisbee Delicious apple fruits. Journal of agricultural and food chemistry. 1991;39: 1902-1906.

21. Lavilla T, Puy J, López ML, Recasens I, Vendrell M. Relationships between Volatile Production, Fruit Quality, and Sensory Evaluation in Granny Smith Apples Stored in Different Controlled-Atmosphere Treatments by Means of Multivariate Analysis. Journal of Agricultural \& Food Chemistry. 1999;47: 3791-3803.

22. López ML, Lavilla T, Recasens I, Riba M, Vendrell M. Influence of Different Oxygen and Carbon Dioxide Concentrations during Storage on Production of Volatile Compounds by Starking Delicious Apples. J Agric Food Chem. 1998;46: 634-643.

23. Plotto A, McDaniel MR, Mattheis JP. Characterization of Changes in 'Gala' Apple Aroma during Storage Using Osme Analysis, a Gas Chromatography-Olfactometry Technique. Journal of the American Society for Horticultural Science. 2000;125.

24. Zhang Y, Wang G, Dong J, et al. Analysis of Volatile Components in Strawberry Cultivars 'Xingdu 1' and 'Xingdu 2' and Their Parents. Agricultural Sciences in China. 2009;8: 441-446.

25. Zhu H, Li XP, Yuan RC, Chen YF, Chen WX. Changes in volatile compounds and associated relationships with other ripening events in banana fruit. Journal of Horticultural Science \& Biotechnology. 2010;85: 283-288.

26. Zhu X, Li Q, Li J, Luo J, Chen W, Li X. Comparative Study of Volatile Compounds in the Fruit of Two Banana Cultivars at Different Ripening Stages. Molecules. 2018;23: 2456.

27. Lopez ML, Lavilla MT, Riba M, Vendrell M. Comparison of volatile compounds in two seasons in apples: Golden delicious and Granny smith. Journal of Food Quality. 1998;21: 155-166.

28. Schneider C, Pratt DA, Porter NA, Brash AR. Control of oxygenation in lipoxygenase and cyclooxygenase catalysis. Chemistry \& biology. 2007;14: 473-488.

29. Chen J, Lu J, He Z, Zhang F, Zhang S, Zhang H. Investigations into the production of volatile compounds in Korla fragrant pears (Pyrus sinkiangensis Yu). Food Chem. 2020;302: 125337.

30. Park S, Sugimoto N, Larson MD, Beaudry R, van Nocker S. Identification of genes with potential roles in apple fruit development and biochemistry through large-scale statistical analysis of expressed sequence tags. Plant Physiol. 2006;141: 811-824.

31. Souleyre EJ, Chagne D, Chen X, et al. The AAT1 locus is critical for the biosynthesis of esters contributing to 'ripe apple' flavour in 'Royal Gala' and 'Granny Smith' apples. Plant J. 2014;78: 903915.

32. Hong G-J, Xue X-Y, Mao Y-B, Wang L-J, Chen X-Y. Arabidopsis MYC2 Interacts with DELLA Proteins in Regulating Sesquiterpene Synthase Gene Expression. Plant Cell. 2012;24: 2635-2648.

33. Yu Z, Li J, Yang C, Hu W, Wang L, Chen X. The Jasmonate-Responsive AP2/ERF Transcription Factors AaERF1 and AaERF2 Positively Regulate Artemisinin Biosynthesis in Artemisia annua L. Mol Plant. 2012;5: 353-365. 
34. Zhang Y, Yin X, Xiao Y, et al. An ETHYLENE RESPONSE FACTOR-MYB Transcription Complex Regulates Furaneol Biosynthesis by Activating QUINONE OXIDOREDUCTASE Expression in Strawberry. Plant Physiol. 2018;178: 189-201.

35. Xu YH, Wang JW, Wang S, Wang JY, Chen XY. Characterization of GaWRKY1, a cotton transcription factor that regulates the sesquiterpene synthase gene (+)-delta-cadinene synthase-A. Plant Physiol. 2004;135: 507-515.

36. Shalit M, Katzir N, Tadmor Y, et al. Acetyl-CoA : Alcohol acetyltransferase activity and aroma formation in ripening melon fruits. Journal of agricultural and food chemistry. 2001;49: 794-799.

37. Gambino G, Perrone I, Gribaudo I. A Rapid and effective method for RNA extraction from different tissues of grapevine and other woody plants. Phytochemical Analysis. 2008;19: 520-525.

38. Anders S, Huber W. Differential expression analysis for sequence count data. Genome Biology. 2010;11: 1-12.

39. Mortazavi A, Williams BA, McCue K, Schaeffer L, Wold BJ. Mapping and quantifying mammalian transcriptomes by RNA-Seq. Nature Methods. 2008;5: 621-628.

40. Livak KJ, Schmittgen TD. Analysis of Relative Gene Expression Data Using Real-Time Quantitative PCR and the $2^{-\triangle \Delta C T}$ Method. Methods. 2001;25: 402-408.

41. Yu G, Wang LG, Han Y, He QY. clusterProfiler: an R package for comparing biological themes among gene clusters. Omics : a journal of integrative biology. 2012;16(5):284-287

42. Young, M.D., Wakefield, M.J., Smyth, G.K. et al. Gene ontology analysis for RNA-seq: accounting for selection bias. Genome Bio.2010; 11: R14.

\section{Figures}


A

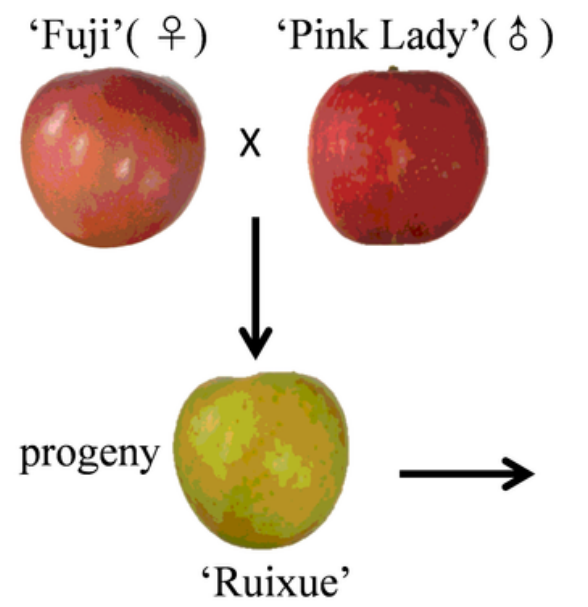

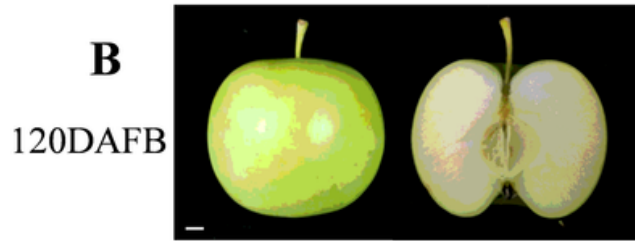

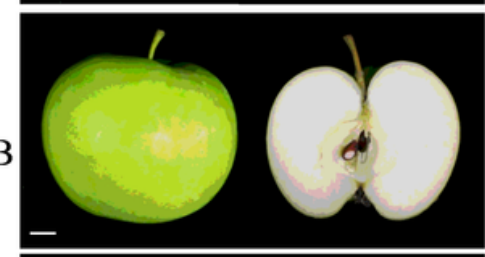

150DAFB

170DAFB

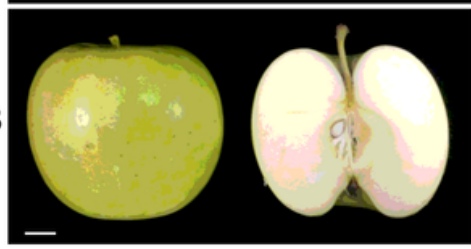

180DAFB
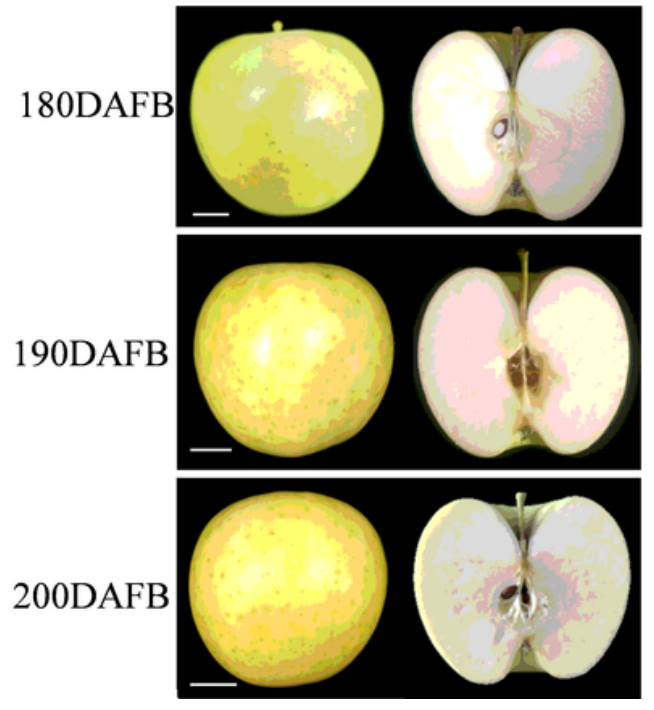

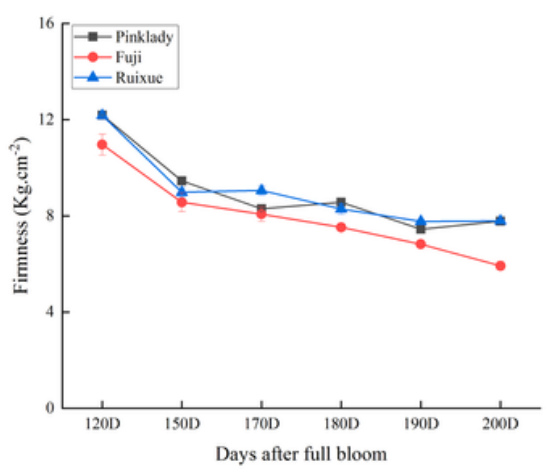

C

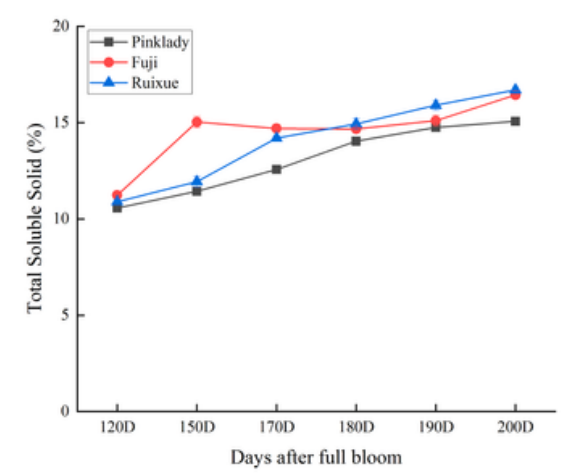

E

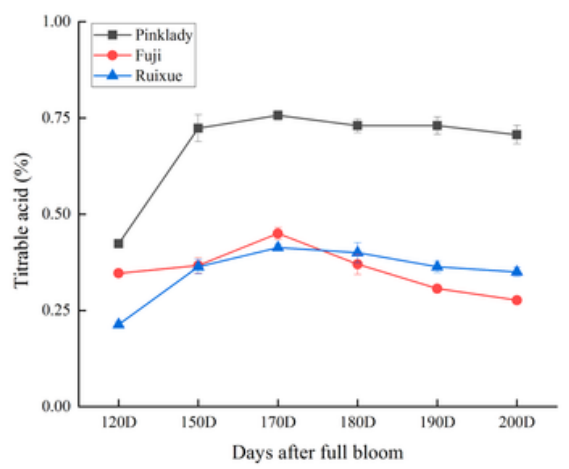

Figure 1

Physiological characters of'Ruixue' and its parents apples during fruit development. (A) Appearance of Ruixue' and its parents apples (B) Appearance of'Ruixue' fruit at various development stages: 120 DAFB, 150 DAFB, 170 DAFB, 180 DAFB, 190 DAFB and 200 DAFB (C) Fruit firmness of'Ruixue' and its parents apples during fruit development. (D) Total soluble solids (TSS) of'Ruixue' and its parents apples during fruit development. (E) Titratable acidity (TA) of'Ruixue' and its parents apples during fruit development. DAFB: days after full bloom, Bars $=1 \mathrm{~cm}$. 
A

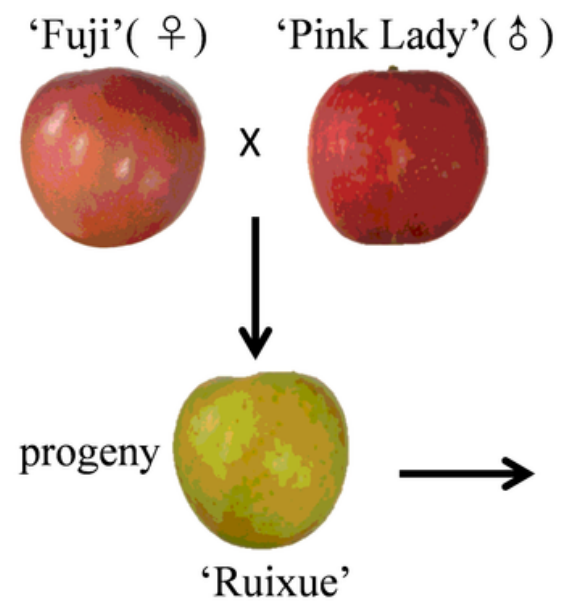

B
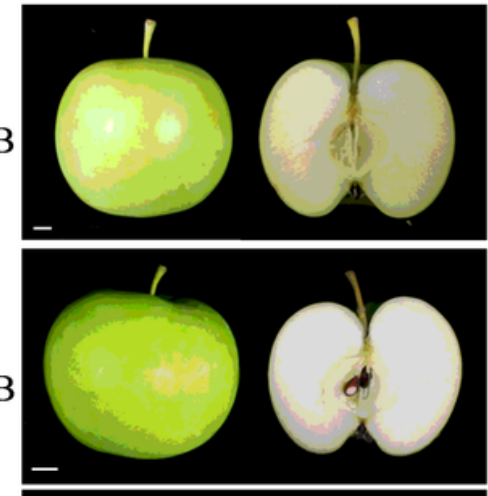

170DAFB

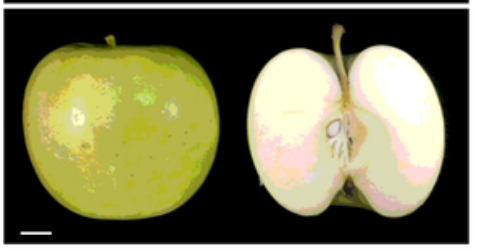

180DAFB
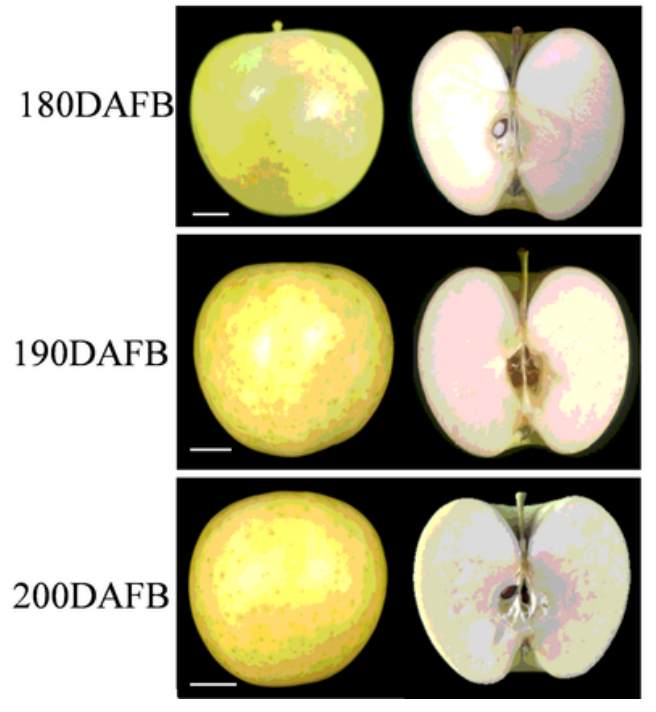

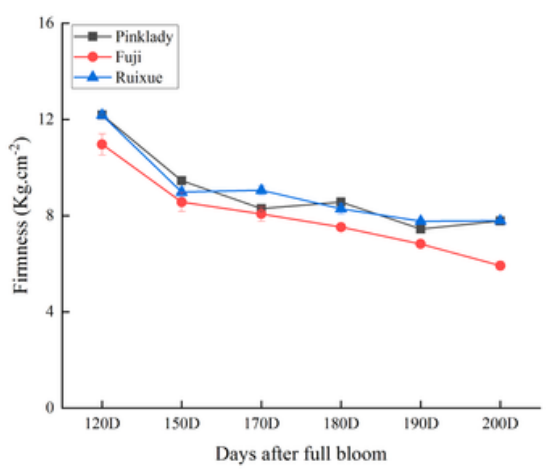

$\mathbf{C}$

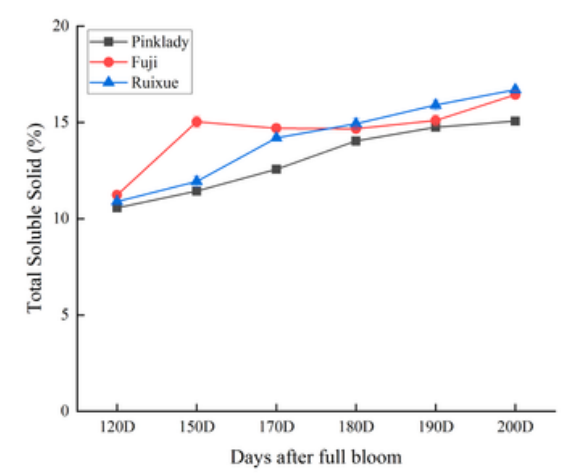

$\mathbf{E}$

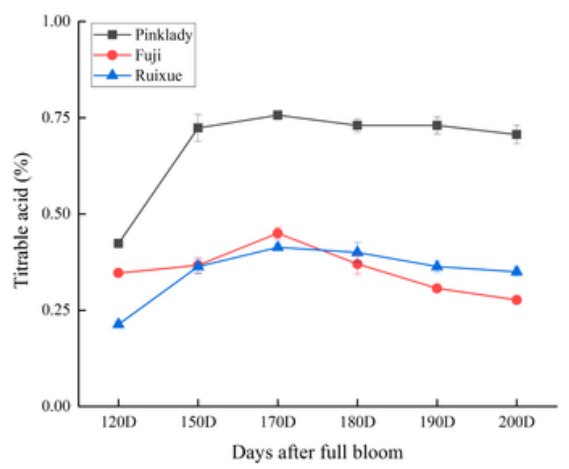

Figure 1

Physiological characters of'Ruixue' and its parents apples during fruit development. (A) Appearance of Ruixue' and its parents apples (B) Appearance of'Ruixue' fruit at various development stages: 120 DAFB, 150 DAFB, 170 DAFB, 180 DAFB, 190 DAFB and 200 DAFB (C) Fruit firmness of'Ruixue' and its parents apples during fruit development. (D) Total soluble solids (TSS) of'Ruixue' and its parents apples during fruit development. (E) Titratable acidity (TA) of'Ruixue' and its parents apples during fruit development. DAFB: days after full bloom, Bars $=1 \mathrm{~cm}$. 
A

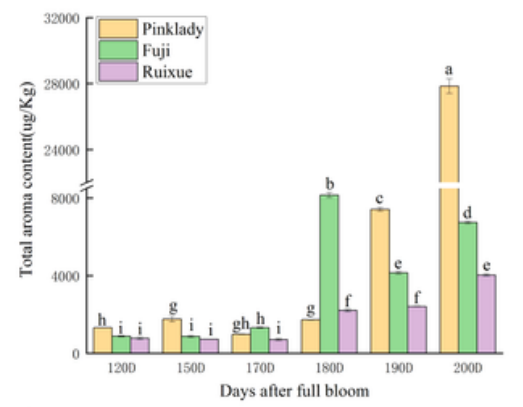

C

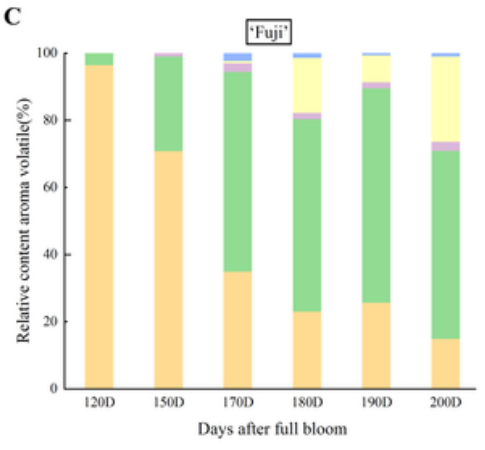

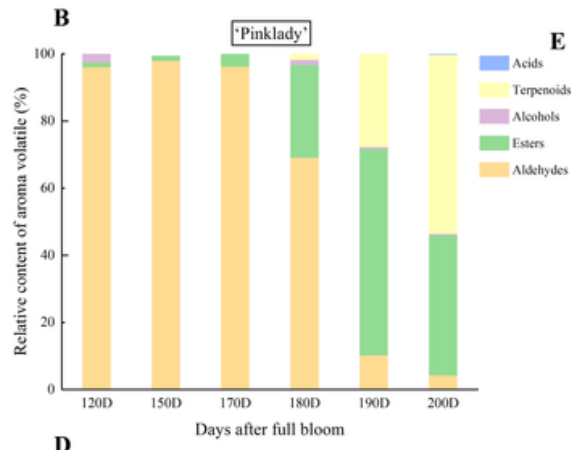

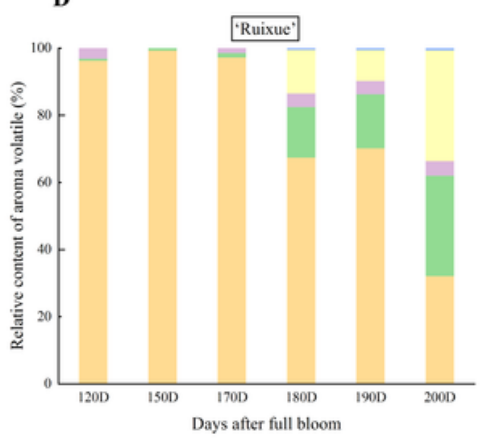

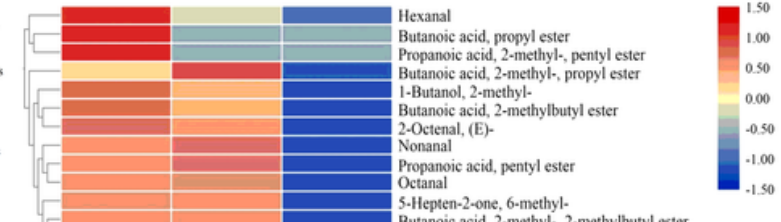

5-Hepten-2-one, 6-methyl-
Butanoic acid, 2-methyl-, 2-methylbutyl ester Propanoic acid, propyl ester Acetic acid propyl ester I-Butanol Butanoic acid, 2-methyl. Propanoic acid, butyl ester
1.Butanol. 2 Heptanoic acid, butyl ester ${ }_{2 \text {-Hexenal }}^{\text {Hetanoli }}$ Hexanoic acid, pentyl ester Hexanoic acid, 2-methylbutyl ester 2-Heptenal, (Z)Butanoic acid, 2-methyl-, pentyl ester Hexanoic acid, hexyl csicr xyl ester Butanoic acid, hexyl ester Isocaryophillene Octanoic acid, hexyl ester Butyl caprylate Propanoic acid, hexyl est Hexanoic acid, butyl ester Acetic acid, butyl ester
Butyl 2-methylbutanoate Butanoic acid, butyl ester 2-Methylbutyl octanoate Hexanoic acid, propyl ester
Acetic acid, hexyl ester Propanoic acid, 2-methyl., hexyl ester

\section{Figure 2}

Aroma profile of'Ruixue' and its parents apples during fruit development. (A) Total aroma content ( $\mu \mathrm{g} / \mathrm{kg}$ ) during fruit development. (B) Relative content of main aroma components in 'Pink Lady' during fruit development. (C) Relative content of main aroma components in 'Fuji' during fruit development. (D) Relative content of main aroma components in'Ruixue' during fruit development. (E) Heatmap with hierarchical clustering of aroma content $(\mu \mathrm{g} / \mathrm{kg})$ in'Ruixue' and its parents apples at 200 DAFB (maturation stage). DAFB: days after full bloom. Error bars show \pm SE from three biological replicates. Significant differences are showed with different letters above the bars $(P<0.05)$.
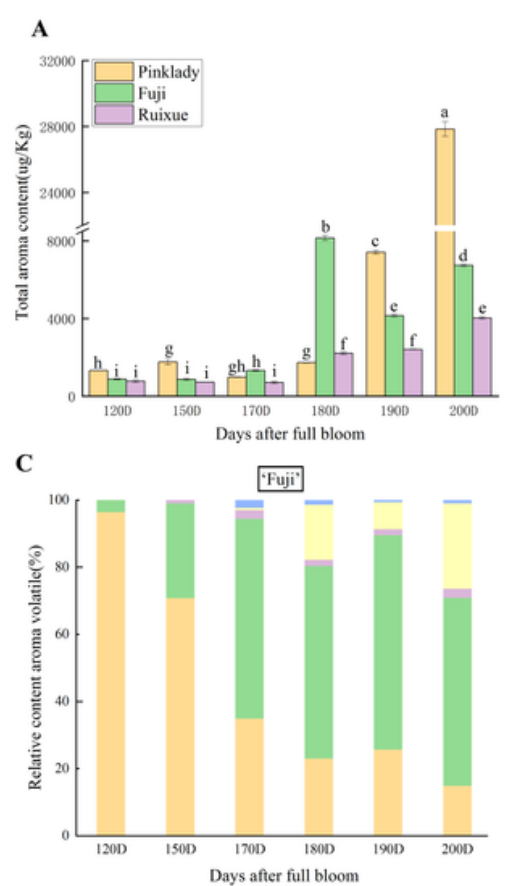
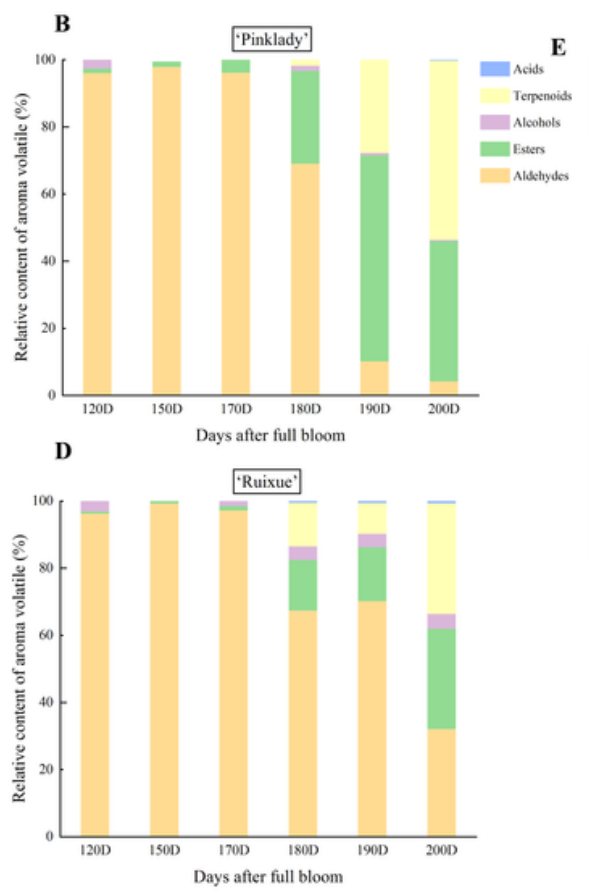

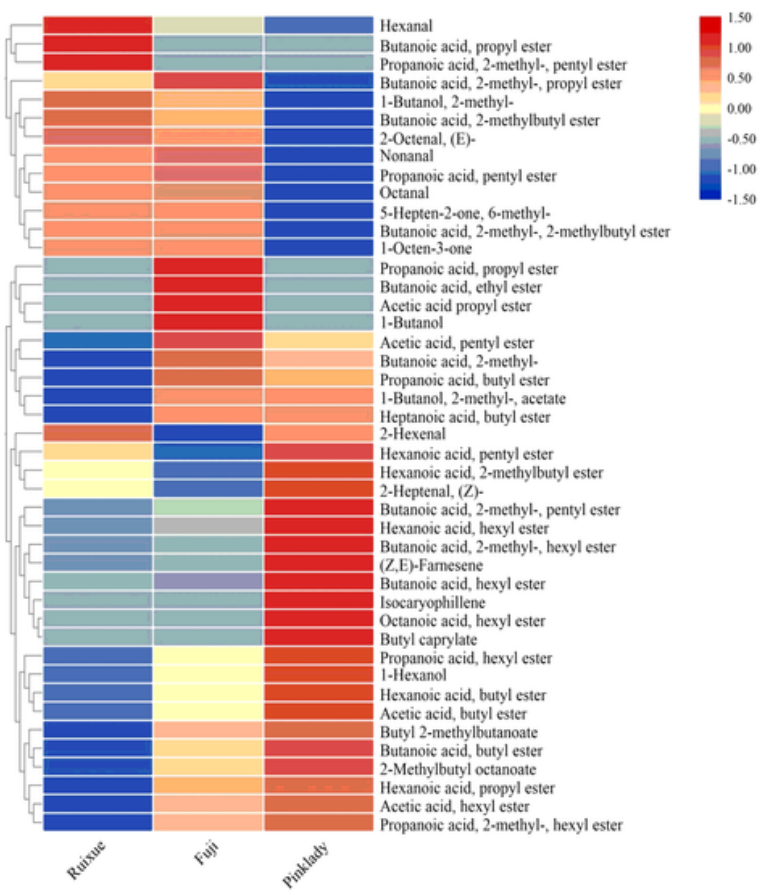


Figure 2

Aroma profile of 'Ruixue' and its parents apples during fruit development. (A) Total aroma content $(\mu \mathrm{g} / \mathrm{kg})$ during fruit development. (B) Relative content of main aroma components in 'Pink Lady' during fruit development. (C) Relative content of main aroma components in 'Fuji' during fruit development. (D) Relative content of main aroma components in'Ruixue' during fruit development. (E) Heatmap with hierarchical clustering of aroma content $(\mu \mathrm{g} / \mathrm{kg})$ in'Ruixue' and its parents apples at 200 DAFB (maturation stage). DAFB: days after full bloom. Error bars show \pm SE from three biological replicates. Significant differences are showed with different letters above the bars $(P<0.05)$.
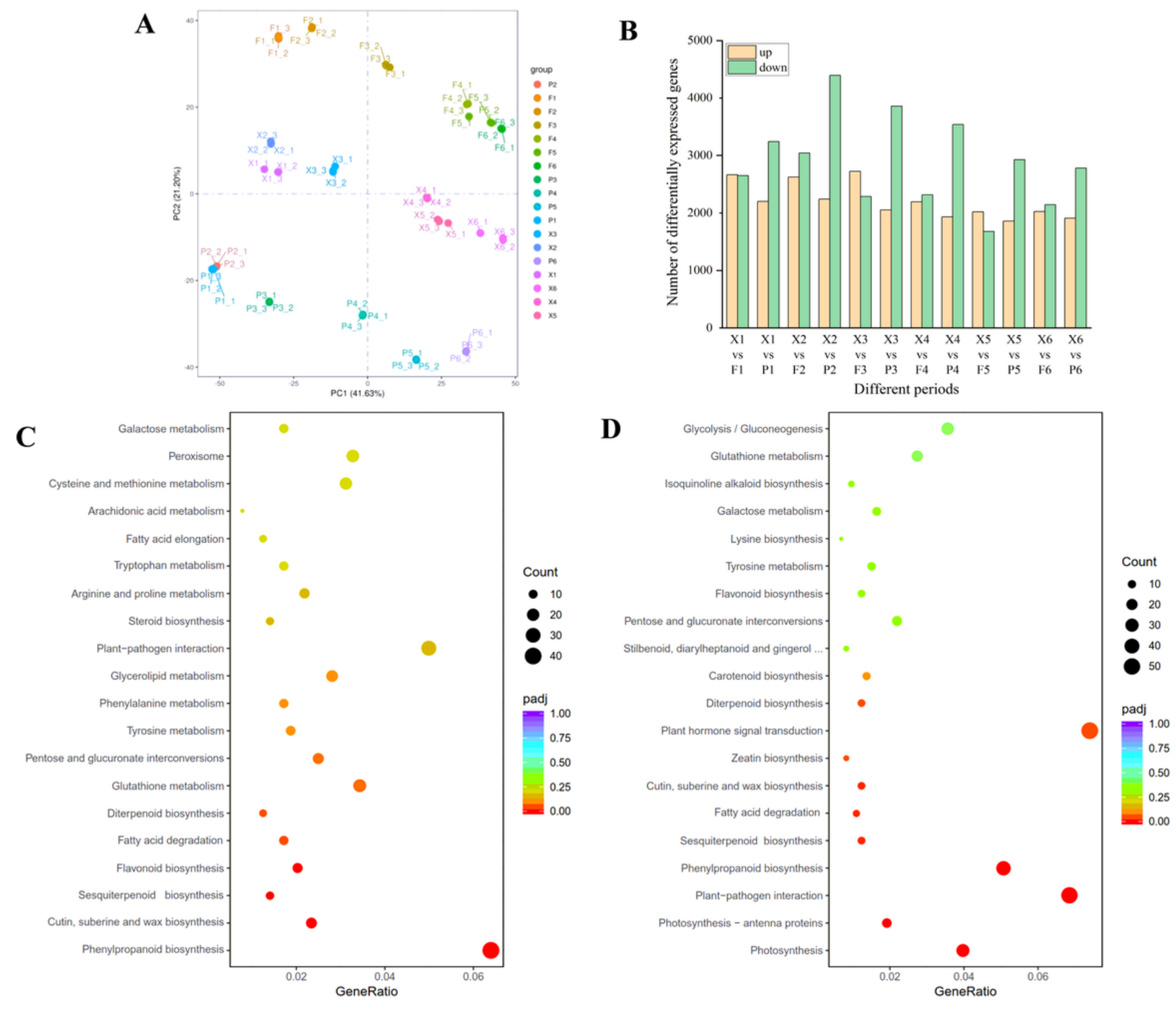

\section{Figure 3}

Summary analysis of transcriptomes. (A) Principal component analysis (PCA) of transcriptome of 54 samples from'Ruixue' and its parents apples during fruit development. (B) Differentially expressed genes 
(DEGs) of'Ruixue' and its parents apples during fruit development. (C) KEGG pathway analysis of'Ruixue' and 'Fuji' at 170 DAFB. (D) KEGG pathway analysis of'Ruixue' and 'Pink Lady' at 170 DAFB. 'F1, F2 F3, F4, F5 and F6" respectively represents at 120, 150, 170, 180, 190 and 200 DAFB(days after full bloom ) of 'Fuji'; “P1, P2, P3, P4, P5 and P6 " respectively represents1 at 120, 150, 170, 180, 190 and 200 DAFB(days after full bloom ) of 'Pink Lady'; "X1, X2, X3, X4, X5 and X6" respectively represents at 120 $, 150,170,180,190$ and 200 DAFB(days after full bloom ) of'Ruixue'.
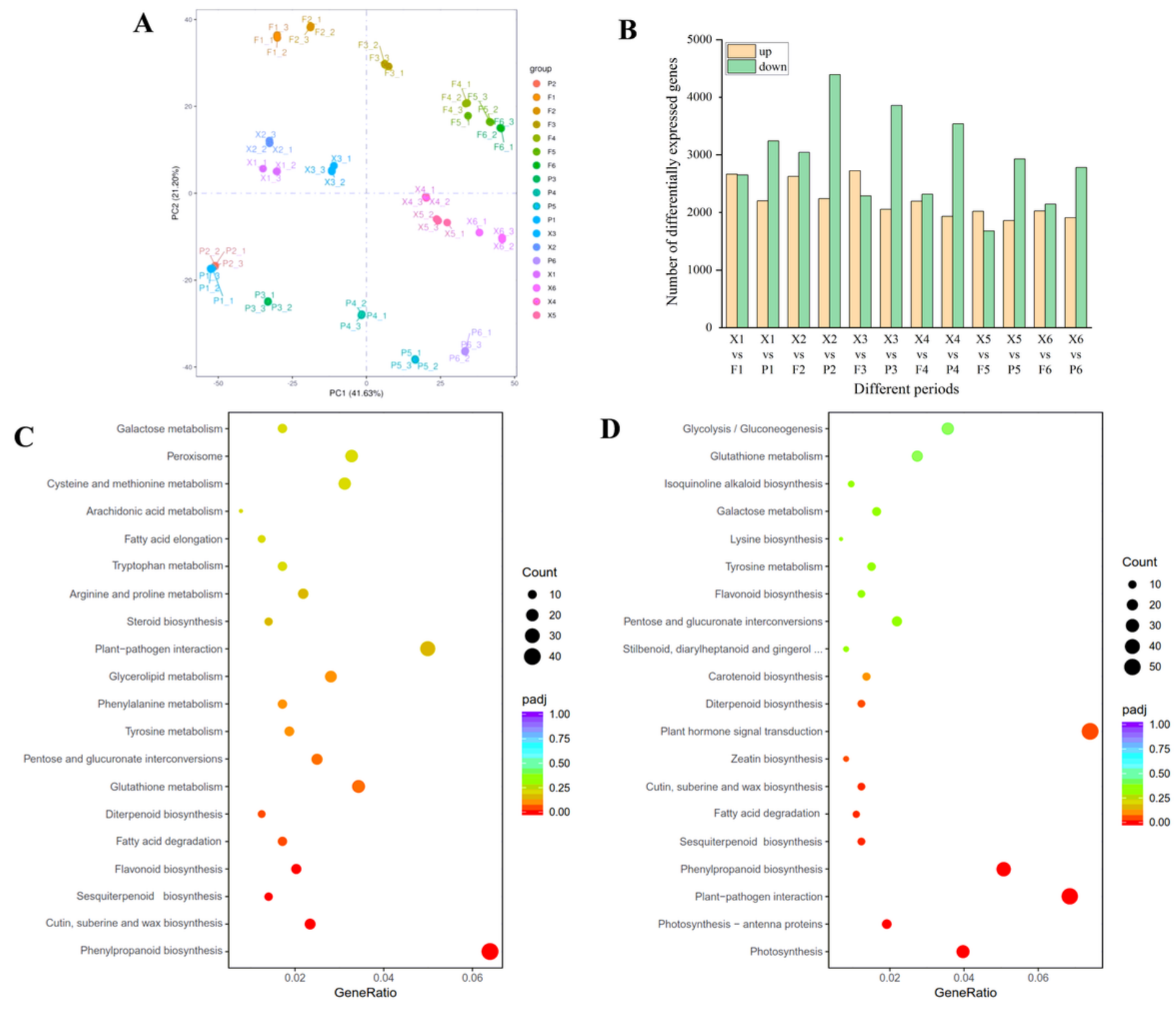

Figure 3

Summary analysis of transcriptomes. (A) Principal component analysis (PCA) of transcriptome of 54 samples from'Ruixue' and its parents apples during fruit development. (B) Differentially expressed genes (DEGs) of'Ruixue' and its parents apples during fruit development. (C) KEGG pathway analysis of'Ruixue' and 'Fuji' at 170 DAFB. (D) KEGG pathway analysis of'Ruixue' and 'Pink Lady' at 170 DAFB. 'F1, F2 F3, F4, F5 and F6" respectively represents at 120, 150, 170, 180, 190 and 200 DAFB(days after full bloom ) of 
'Fuji'; “P1, P2, P3, P4, P5 and P6 " respectively represents1 at 120, 150, 170, 180, 190 and 200 DAFB(days after full bloom ) of 'Pink Lady'; "X1, X2, X3, X4, X5 and X6" respectively represents at 120 $, 150,170,180,190$ and 200 DAFB(days after full bloom ) of'Ruixue'.

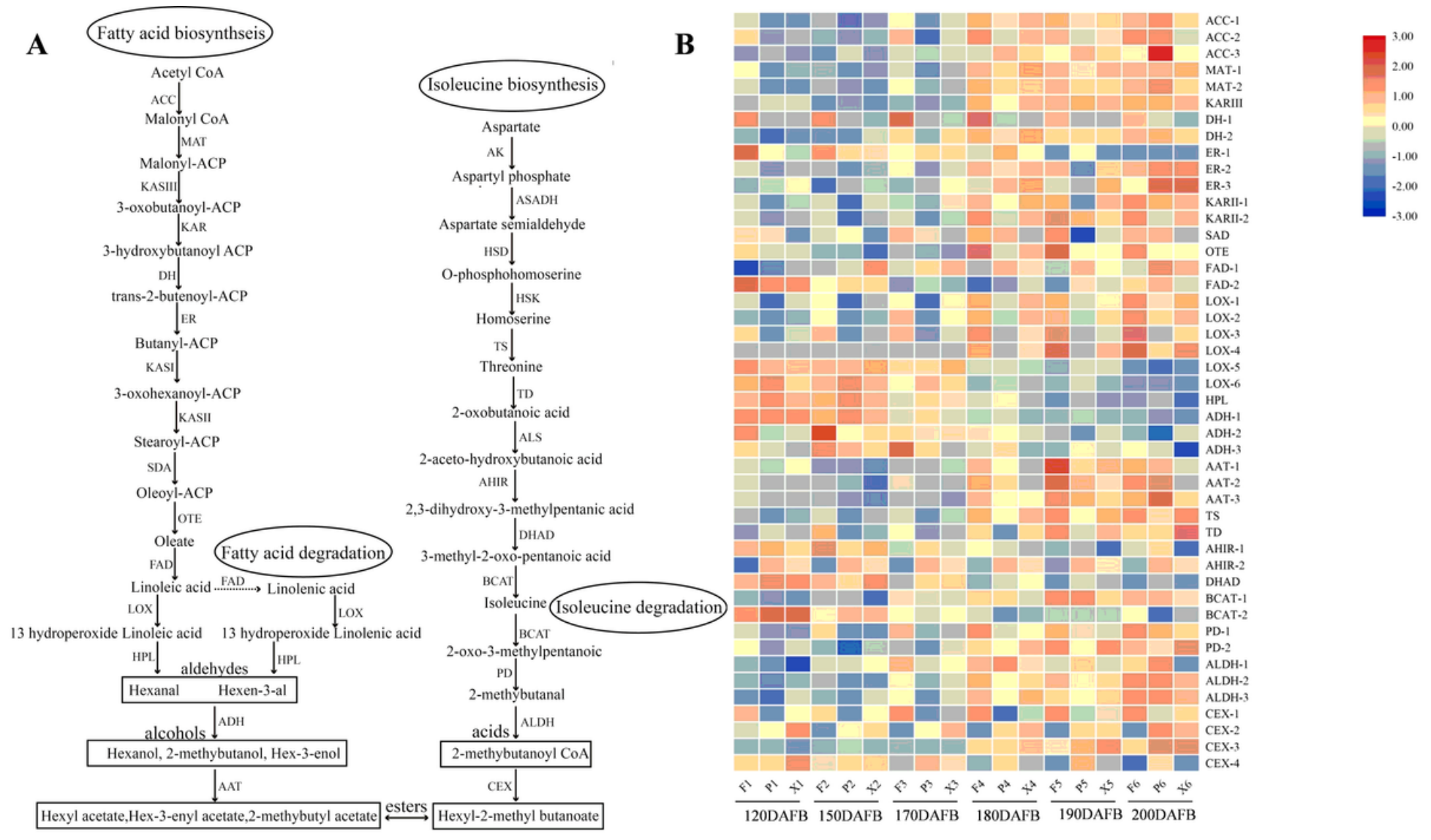

\section{Figure 4}

Expression profiles of aroma-related genes for ester biosynthesis associated with fatty acid and isoleucine pathway during fruit development. (A) Biosynthetic pathway of ester involve in fatty acid and isoleucine pathway. (B) Expression profiles of aroma-related genes for ester biosynthesis. ACC: AcetylCoA carboxylase; MAT: Malonyl-CoA ACP transacylase; KASIII: 3-Ketoacyl ACP synthase III; KAR: 3Ketoacyl ACP reductase; DH: 3-Hydroxyacyl ACP dehydratase; ER: 2,3-Trans-enoyl ACP reductase; KASI: 3Ketoacyl ACP synthase I; KASII: 3-Ketoacyl ACP synthase II; SAD: Stereate ACP desaturase; OTE: OleateACP thioesterase; FAD: Oleate and linoleate desaturase; AK: Aspartate kinase; ASADH: Aspartate semialdehyde dehydrogenase; HSD: Homoserine dehydrogenase; HSK: Homoserine kinase; TS: Threonine synthase; TD: Threonine deaminase; ALS: Acetolactic synthetase; AHIR: Acetohydroacid isomeroreductase; DHAD: Dihydroxy acid dehydratase; BCAT: Branched chain aminotransferase; LOX: Lipoxygenase; HPL: Hydroperoxide lyase; PD: Pyruvate decarboxylase; ADH: Alcohol dehydrogenase; ALDH: Aldehyde dehydrogenase; AAT: Alcohol acyl transferase; CXE: Carboxylesterase. 

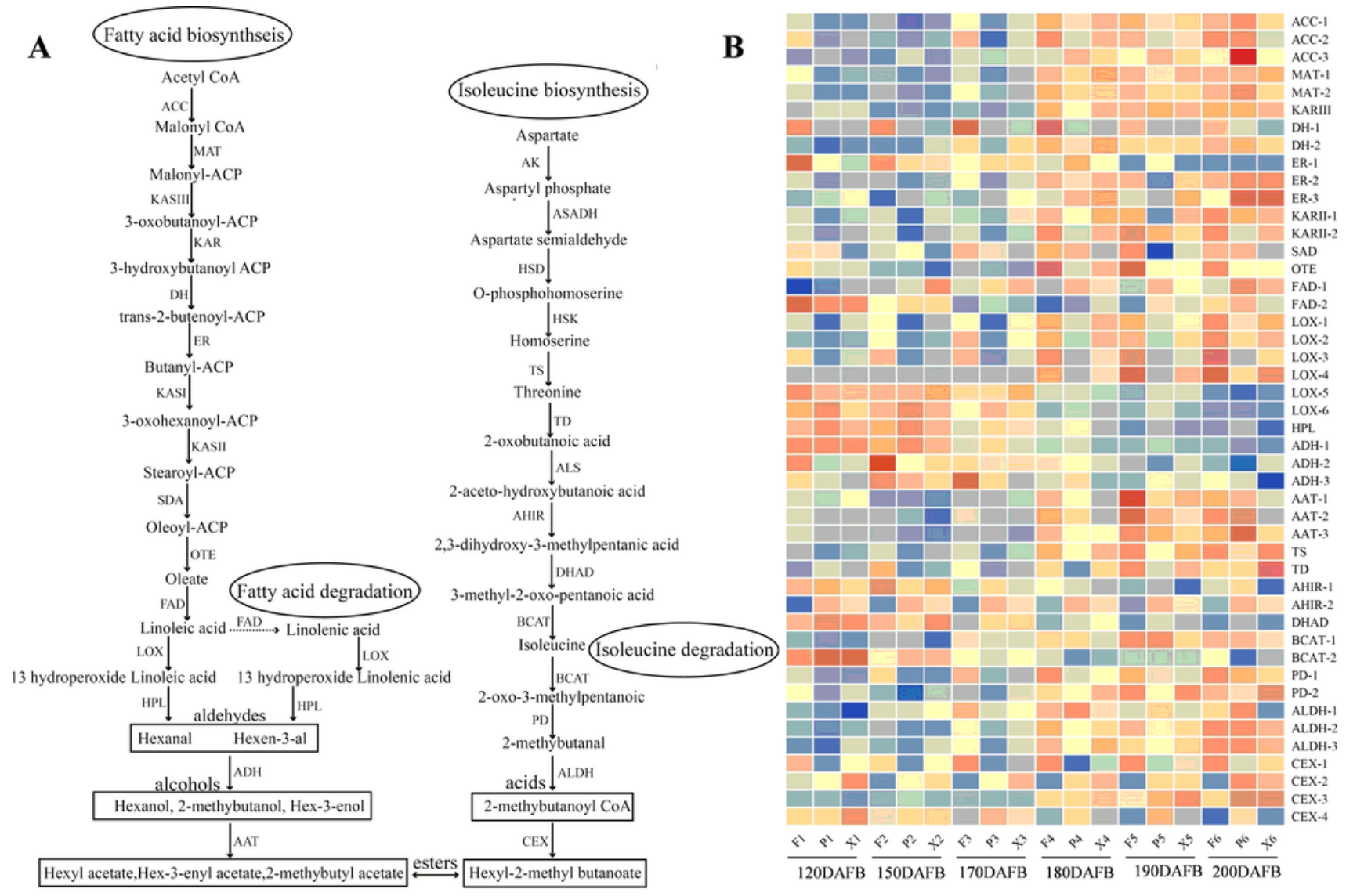

\section{Figure 4}

Expression profiles of aroma-related genes for ester biosynthesis associated with fatty acid and isoleucine pathway during fruit development. (A) Biosynthetic pathway of ester involve in fatty acid and isoleucine pathway. (B) Expression profiles of aroma-related genes for ester biosynthesis. ACC: AcetylCoA carboxylase; MAT: Malonyl-CoA ACP transacylase; KASIII: 3-Ketoacyl ACP synthase III; KAR: 3Ketoacyl ACP reductase; DH: 3-Hydroxyacyl ACP dehydratase; ER: 2,3-Trans-enoyl ACP reductase; KASI: 3Ketoacyl ACP synthase I; KASII: 3-Ketoacyl ACP synthase II; SAD: Stereate ACP desaturase; OTE: OleateACP thioesterase; FAD: Oleate and linoleate desaturase; AK: Aspartate kinase; ASADH: Aspartate semialdehyde dehydrogenase; HSD: Homoserine dehydrogenase; HSK: Homoserine kinase; TS: Threonine synthase; TD: Threonine deaminase; ALS: Acetolactic synthetase; AHIR: Acetohydroacid isomeroreductase; DHAD: Dihydroxy acid dehydratase; BCAT: Branched chain aminotransferase; LOX: Lipoxygenase; HPL: Hydroperoxide lyase; PD: Pyruvate decarboxylase; ADH: Alcohol dehydrogenase; ALDH: Aldehyde dehydrogenase; AAT: Alcohol acyl transferase; CXE: Carboxylesterase. 
A

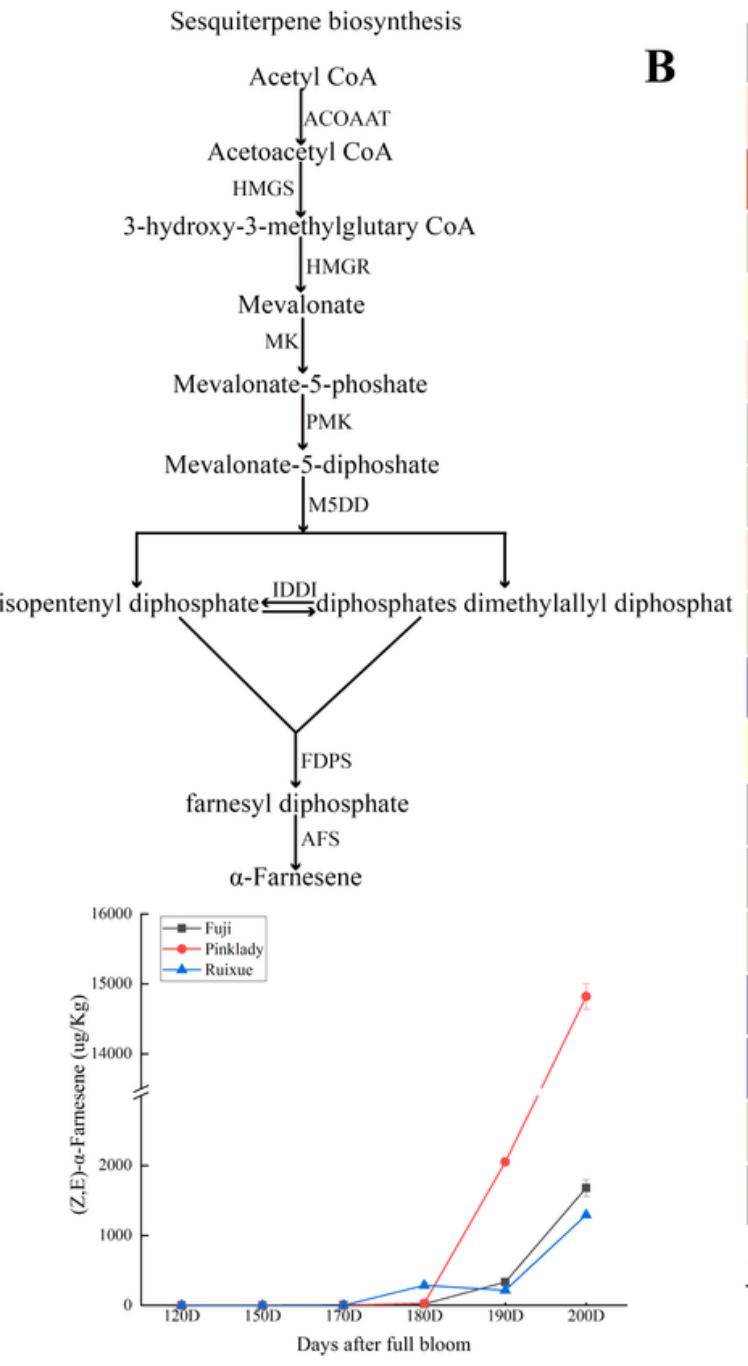

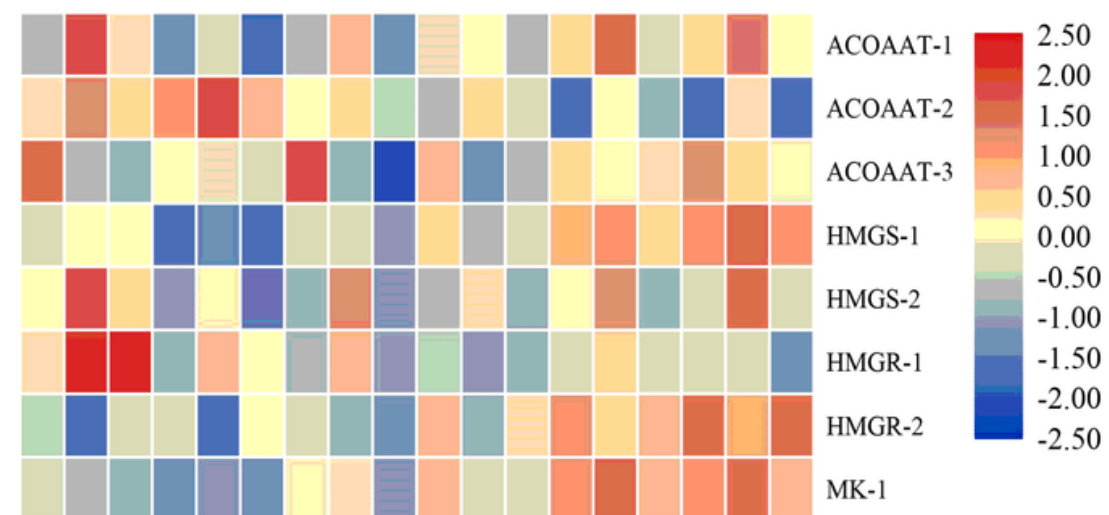

MK-2

PMK

M5DD- 1

M5DD-2

IDDI-1

IDDI-2

FDPS-1

FDPS-2

FDPS-3

FDPS-4

AFS

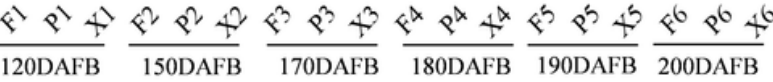

\section{Figure 5}

Expression profiles of aroma-related genes for sesquiterpenes biosynthetic pathway during fruit development. (A) Biosynthetic pathway of sesquiterpenes. (B) Expression profiles of aroma-related genes for sesquiterpenes biosynthesis. ACOAAT: Acetyl-CoA acetyltransferase; HMGS: HMG-CoA synthase; HMGR: HMG-CoA reductase; MK: Melalonate kinase; PMK: Phosphomevalonate kinase; M5DD: Mevalonate 5-diphosphate decarboxylase; IDDI: Isopentyl-diphosphate $\Delta$-isomerase; PPS: Polyprenyl synthase; AFS: a-Farnesene synthase. (C) The change in hexanol of Ruixue' and its parents apples during fruit development. 
A

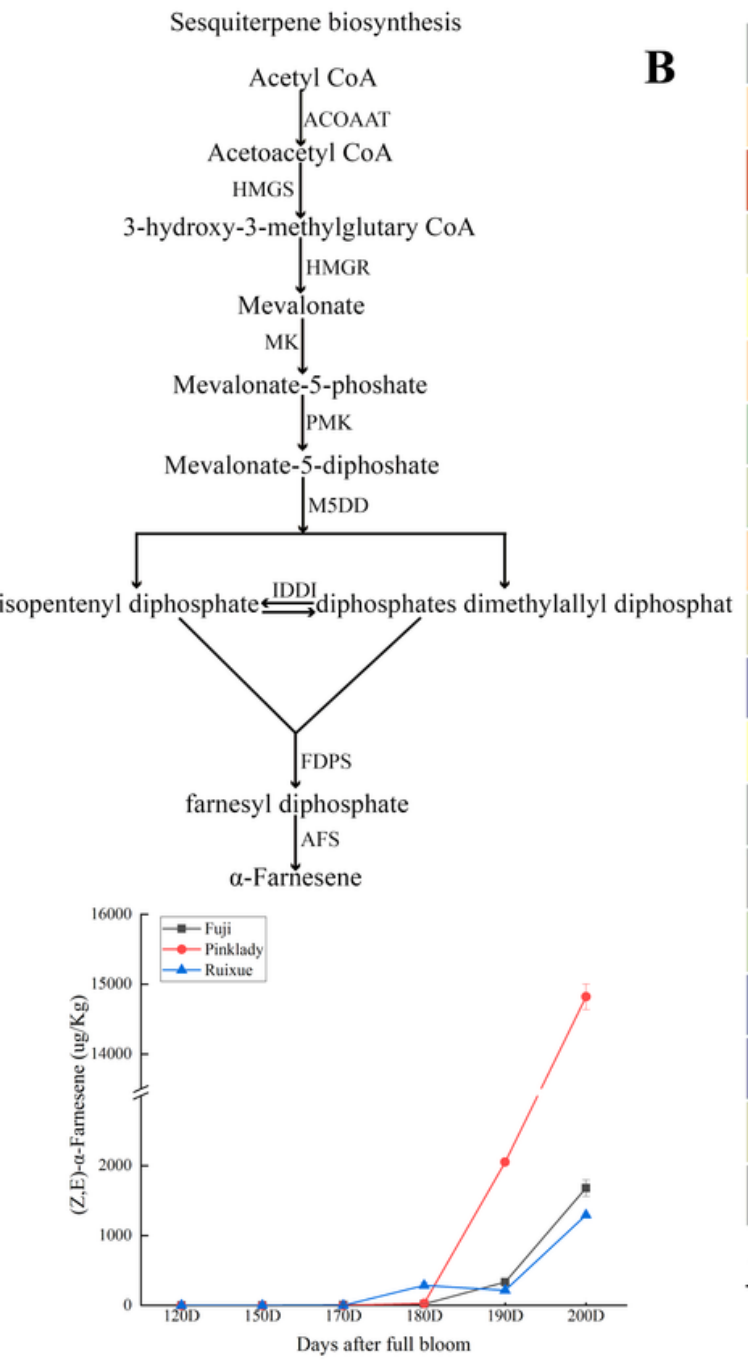

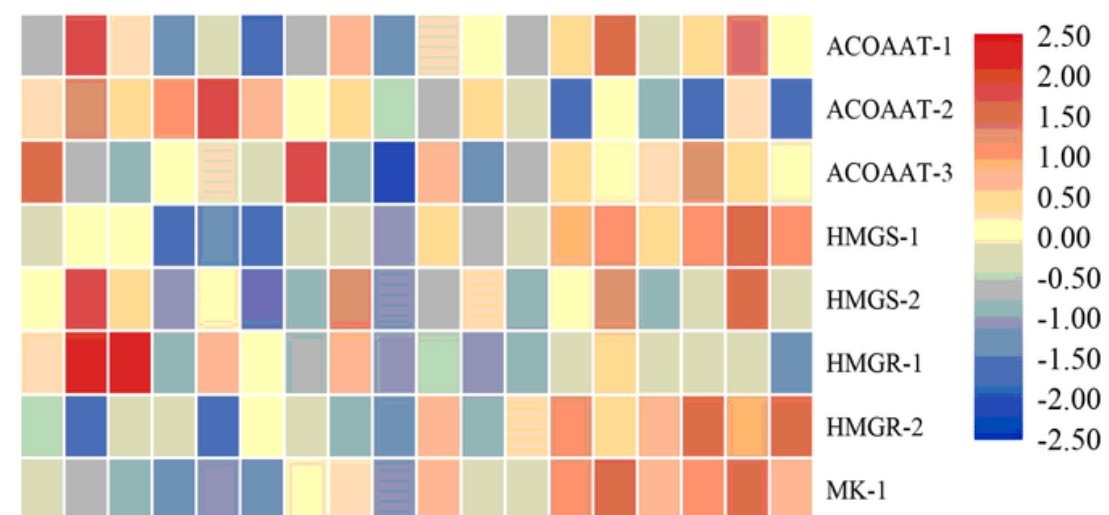

MK-2

PMK

M5DD-1

M5DD-2

IDDI-1

IDDI-2

FDPS-1

FDPS-2

FDPS-3

FDPS-4

AFS

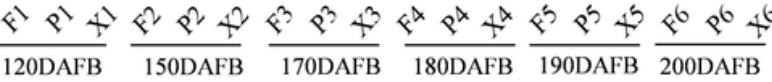

\section{Figure 5}

Expression profiles of aroma-related genes for sesquiterpenes biosynthetic pathway during fruit development. (A) Biosynthetic pathway of sesquiterpenes. (B) Expression profiles of aroma-related genes for sesquiterpenes biosynthesis. ACOAAT: Acetyl-CoA acetyltransferase; HMGS: HMG-CoA synthase; HMGR: HMG-CoA reductase; MK: Melalonate kinase; PMK: Phosphomevalonate kinase; M5DD: Mevalonate 5-diphosphate decarboxylase; IDDI: Isopentyl-diphosphate $\Delta$-isomerase; PPS: Polyprenyl synthase; AFS: a-Farnesene synthase. (C) The change in hexanol of Ruixue' and its parents apples during fruit development. 

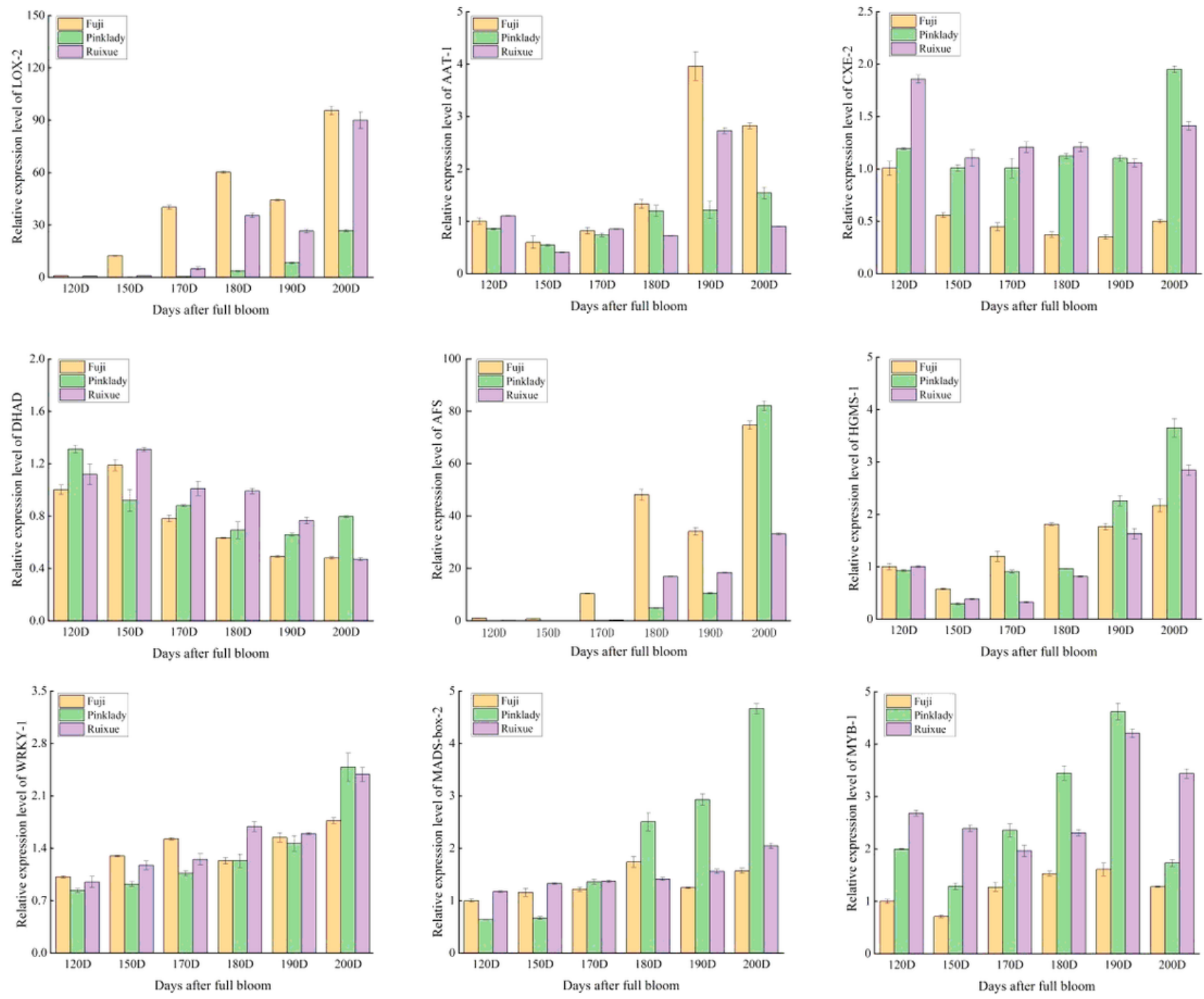

Figure 6

qRT-PCR validated candidate genes in Ruixue' and its parents apples during fruit development. Error bars show \pm SE from three biological replicates 

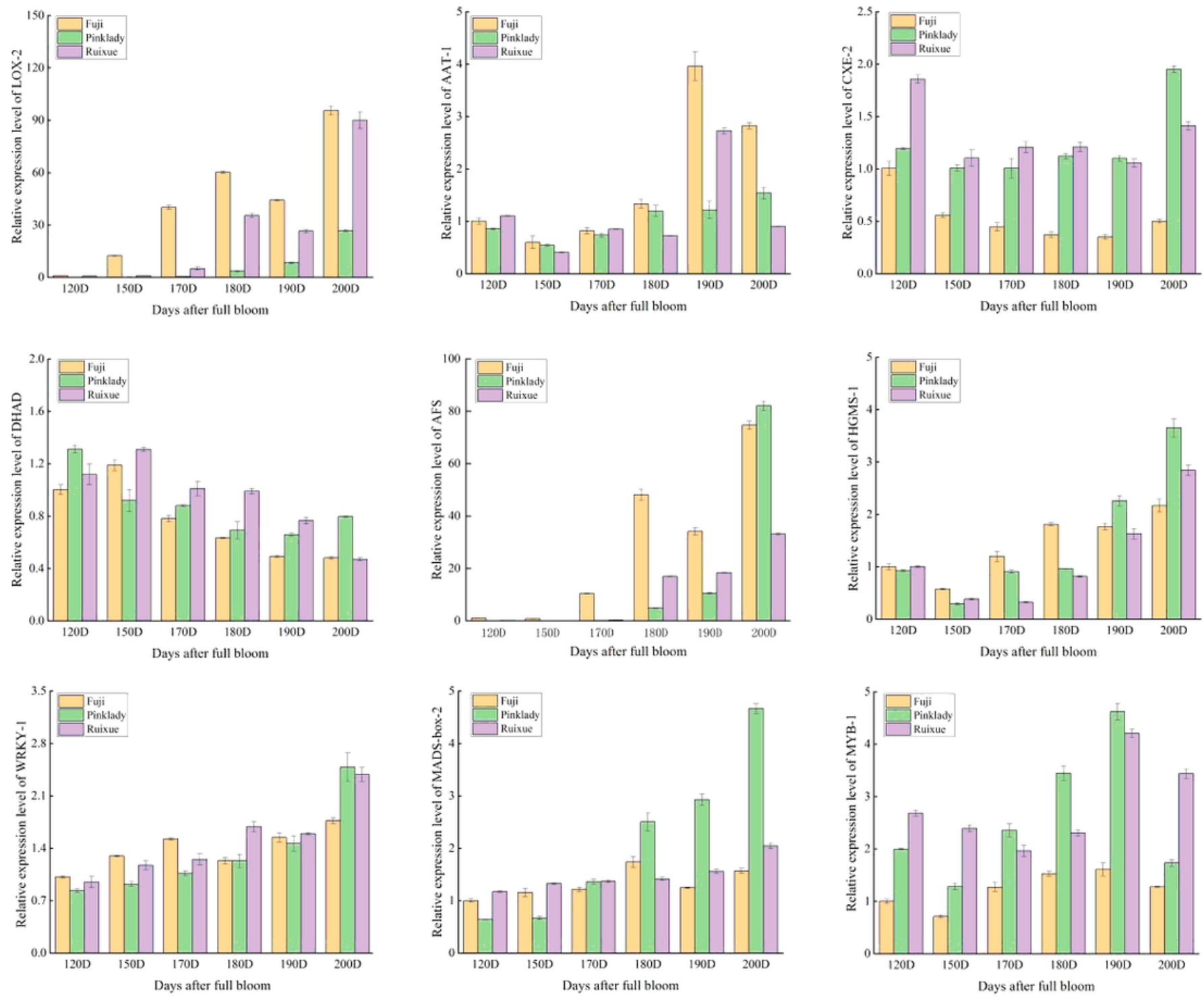

Figure 6

qRT-PCR validated candidate genes in Ruixue' and its parents apples during fruit development. Error bars show \pm SE from three biological replicates

\section{Supplementary Files}

This is a list of supplementary files associated with this preprint. Click to download.

- SuppFig.Png

- SuppFig.Png

- AppendixA.Supplementarydata.pdf

- AppendixA.Supplementarydata.pdf 